\title{
Linking the Kar and Folk Solutions Through a Problem Separation Property
}

\author{
Christian Trudeau (University of Windsor)
}

Working paper $13-01$

Working papers are in draft form. This working paper is distributed for purposes of comment and discussion only. It may not be reproduced without permission of the copyright holder. Copies of working papers are available from the author or at http://ideas.repec.org/s/wis/wpaper.html. 


\title{
Linking the Kar and Folk Solutions Through a Problem Separation Property
}

\author{
Christian Trudeau*
}

This draft: October 2012

\begin{abstract}
Minimum cost spanning tree problems connect agents efficiently to a source with the cost of using an edge fixed. We revisit the dispute between the Kar and folk solutions, two solution concepts to divide the common cost of connection based on the Shapley value. We introduce a property called Weak Problem Separation that allows, under conditions, to divide the problem in two: connecting an agent to the source and connecting agents to each other. It allows us to characterize the set of all affine combinations of the Kar and folk solutions.

Keywords: Minimum cost spanning tree problems; folk solution; Kar solution; problem separability.

JEL Classifications: C71, D63
\end{abstract}

\section{Introduction}

Minimum cost spanning tree (mcst) problems study situations where a group of agents, located at different points in space, need to be connected to a source. Agents can be connected directly to this source or indirectly through other agents already connected. Connection costs on an edge between two agents or between an agent and the source is a fixed cost, invariant with the number of users connecting through it. Examples of economic situations that can be modeled as mcst problems include electricity distribution networks as well as communication networks such as Internet, cable TV or telephone.

The literature on cost sharing solutions to the mcst problem is well established (see Trudeau (2012a)). The two main cost sharing solutions are built around the familiar Shapley value. Kar (2002) applies the Shapley value to the stand-alone game associated with the mcst problem, where a coalition can only use edges between its agents to build an optimal network. This solution is known as the Kar solution. The second method was discovered independently in Feltkamp et al (1994) and Bergantinos and Vidal-Puga (2007a), as well as being the average of the family of solutions proposed in Norde et al (2004). While there are many ways to interpret the solution, one of them consists in defining the irreducible cost matrix, which is such that the costs of edges on the mcst remain unchanged, while the costs on other edges are reduced up to the point where further reductions would change the total cost to connect everybody to the source. The so-called folk solution is the Shapley value of the stand-alone game associated with the irreducible cost matrix. Remarkably, the two solutions have not been characterized together. However, Bergantinos and Vidal-Puga (2010) show that they can be implemented using similar non-cooperative mechanisms. Families of solutions that include the folk solution have been characterized, but they do not include the Kar solution (Bergantinos and Kar (2010)).

The Kar solution has been criticized in the recent literature for two reasons: it does not always propose a stable solution where no coalition has an incentive to quit the group and do the project on its own, and it sometimes proposes negative cost shares.

${ }^{*}$ Department of Economics, University of Windsor

401 Sunset Avenue, Windsor, Ontario, Canada. Email: trudeauc@uwindsor.ca 
While the first criticism is valid, we feel that the second one is not. In mcst problems, when an agent $i$ joins a coalition $S$, if we suppose that a coalition can only use the location of its members, then it is possible for the cost of connecting agents in $S \cup\{i\}$ to the source to be smaller than the cost to connect agents in $S$. It is natural to impose non-negativity of the cost shares for monotonic games since we do not want agents to pay less than their smallest incremental cost, which are all non-negative. However, for non-monotonic games this natural lower bound is not appropriate as incremental costs can be negative. The assumption that a coalition can only use the locations of its members is natural when there is a notion of property rights over these locations. This is the case in many applications of the mcst model. For example, Russian natural gas producer Gazprom sends gas to Europe through Ukraine. In exchange for allowing European nations to reach the source of natural gas cheaply, Ukraine is compensated with transit fees by Gazprom. Negotiations over these fees have played a role in the many disputes between Ukraine and Gazprom over the past years.

While the folk solution offers a stable and non-negative allocation, it depends only on the irreducible cost matrix. Therefore, a large portion of the information contained in the cost matrix is lost. See Bogomolnaia and Moulin (2010) for a critique of this Reductionism property.

This paper offers a way to reconcile the two cost sharing solutions by defining and characterizing a family of solutions that contains both solutions. This is done by introducing some new properties, with the main one being the Weak Problem Separation property. Suppose that the optimal way to connect agents is to have only one of them connected to the source, and that this edge is the most expensive of the minimum cost spanning tree. Then, Weak Problem Separation says that we can split the problem in two: finding who to connect to the source and then connecting all agents together. Applying a cost sharing solution on the mcst problem or separately on these two problems should yield the same cost shares. This property allows one to split the problem into two simpler problems and is, thus, similar in nature to many properties found in the cost sharing literature, most notably the Additivity property.

This property is combined with three known properties: Piecewise Linearity, Group Independence and Anonymity; and two new ones: Independence of Irrelevant Edges and Weak Equal Treatment. Piecewise Linearity allows one to decompose the problem into elementary problems where edges have a cost of 0 or 1 . Group Independence says that if two groups are such that no pair of agents from different groups gain anything from being connected directly together, then we can share cost independently on each group. Anonymity says that cost shares should not depend on the names of the agents. Independence of Irrelevant Edges states that if the cost of the edge between $i$ and $j$ is larger than the cost to connect $i$ to the source and the cost to connect $j$ to the source, then cost shares should not depend on the cost of the edge between $i$ and $j$, since this edge will never be used. In Kar (2002), Equal Treatment, that says that $i$ and $j$ should be equally responsible for the cost of edge $(i, j)$, is used to characterize the Kar solution. We use a weaker version that applies only to edges that are not in any mest.

The set of solutions satisfying these six properties is composed of solutions that are a weighted sum of the Kar and folk solutions. Since the weights sum to one, we actually have an affine combination of the Kar and folk solutions, leaving only one parameter free. This parameter turns out be the cost share in a simple two-agent problem. This not only shows clearly the difference between the two solutions, but it can also ease implementation. Once agents agree that the six properties are desirable, their opinion of what should be the allocations in the simple two-agent problem will be enough to generate the corresponding cost-sharing solution.

We offer new characterizations of the folk and Kar solutions. The folk solution is obtained by adding Core Selection, that assures that all cost shares are stable allocations. The Kar solution is obtained by strengthening the Weak Problem Separation property.

The structure of the paper is as follows. In section 2 we formally define mcst problems as well as the Kar and the folk solutions. Definitions of the main properties used in the paper are given in section 3 together with the main theorem describing the family of solutions characterized by these properties. Section 4 offers characterizations of the Kar and folk solutions. Some supporting lemmas are in the appendix. 


\section{The setting}

\subsection{Minimum cost spanning tree problems}

Let $\mathcal{N}=\{1,2, \ldots\}$ be the set of potential participants and $N \subseteq \mathcal{N}$ be the finite set of agents that actually need to be connected to the source, denoted by 0 . Let $N_{0}=N \cup\{0\}$. For any set $Z$, define $Z^{p}$ as the set of all non-ordered pairs $(i, j)$ of elements of $Z$. In our context, any element $(i, j)$ of $N_{0}^{p}$ represents the edge between $i$ and $j$. Let $c=\left(c_{e}\right)_{e \in N_{0}^{p}}$ be a vector in $\mathbb{R}_{+}^{N_{0}^{p}}$ with $c_{e}$ representing the cost of edge $e$. Let $\Gamma(N)$ be the set of all cost vectors when the set of agents is $N$. Let $\Gamma$ be the set of all cost vectors, for all possible $N$. Since $c$ assigns a cost to all edges $e$, we often abuse language and call $c$ a cost matrix. A minimum cost spanning tree problem is a triple $(0, N, c)$. Since 0 does not change, we omit it in the following and simply identify an mest problem as $(N, c)$, with $N \subseteq \mathcal{N}$ and $c \in \Gamma(N)$.

A cycle $p_{l l}$ is a set of $K \geq 3$ edges $\left(i_{k}, i_{k+1}\right)$, with $k=0,1, \ldots, K-1$ and such that $i_{0}=i_{K}=l$ and $i_{1}, \ldots, i_{K-1}$ distinct and different than $l$. A path $p_{l m}$ between $l$ and $m$ is a set of $K$ edges $\left(i_{k}, i_{k+1}\right)$, with $k=0,1, \ldots, K-1$, containing no cycle and such that $i_{0}=l, i_{K}=m$ and $i_{1}, \ldots, i_{K-1}$ distinct and different than $l$ and $m$. Let $P_{l m}\left(N_{0}\right)$ be the set of all such paths between $l$ and $m$. For a set of edges $E \subseteq N_{0}^{p}$, we say that $E$ is in $S \subseteq N_{0}$ if for all $(i, j) \in E, i, j \in S$. We say that $E$ contains a cycle in $S$ if, for all $i \in S$, there exists a cycle $p_{i i}$ in $S$ such that all elements of $p_{i i}$ are also in $E$. We say that a path $p_{l m}$ is a free path if $c_{e}=0$ for all $e \in p_{l m}$.

A spanning tree is a non-orientated graph without cycles that connects all elements of $N_{0}$. A spanning tree $t$ is identified by the set of its edges. Its associated cost is $\sum_{e \in t} c_{e}$.

The minimum cost of connecting $N$ to the source and the associated minimum cost spanning tree is obtained using Prim's algorithm, which has $|N|$ steps. First, pick an edge $(0, i)$ such that $c_{0 i} \leq c_{0 j}$ for all $j \in N$. We then say that $i$ is connected. In the second step, we choose an edge with the smallest cost connecting an agent in $N \backslash\{i\}$ either directly to the source or to $i$, which is connected. We continue until all agents are connected, at each step connecting an agent not already connected to an agent already connected or to the source. Let $C(N, c)$ be the associated cost. Note that the mcst might not be unique. Let $t^{*}(c)$ be a minimum cost spanning tree for the cost matrix $c$. Let $T^{*}(c)$ be the set of all minimum cost spanning trees for the cost matrix $c$.

For any $S \subset N$, let $c^{S}$ be the restriction of the cost matrix $c$ to the coalition $S_{0}=S \cup\{0\}$. Let $C(S, c)$ be the cost of an mcst of the problem $\left(S, c^{S}\right)$. Given these definitions, we say that $C$ is the stand-alone cost function associated with $c$.

\subsection{Cost sharing solutions}

For a problem $(N, c)$, a cost allocation $y \in \mathbb{R}^{N}$ assigns a cost share to each agent such that the budget balance condition $\sum_{i \in N} y_{i}=C(N, c)$ is satisfied. Note that these cost shares can be negative. Since $C$ is not necessarily monotonic, we have justification to subsidize an agent.

A cost sharing solution (or rule) assigns a cost allocation $y(N, c)$ to any mcst problem $(N, c)$. We introduce the two solutions that are the focus of the paper.

The Kar solution was explicitly defined and characterized in Kar (2002). It is the Shapley value of the game $C$. More precisely,

$$
y_{i}^{k}(N, c)=S h_{i}(C)=\sum_{S \subseteq N \backslash\{i\}} \frac{|S| !(|N|-|S|-1) !}{|N| !}(C(S \cup\{i\}, c)-C(S, c))
$$

for all $i \in N$, with $C(\emptyset, c)=0$. See Winter (2002) for a review of the broad applications and appeal of the Shapley value.

As mentioned in the introduction, the so-called folk solution has been obtained in different ways. We focus on the approach of Bergantinos and Vidal-Puga (2007a), which uses the Shapley value, thus allowing a clear comparison with the Kar solution. 
From any cost matrix $c$, we can define the irreducible cost matrix $c^{*}$ as follows:

$$
c_{i j}^{*}=\min _{p_{i j} \in P_{i j}\left(N_{0}\right)} \max _{e \in p_{i j}} c_{e} .
$$

The folk solution is the Shapley value of the stand-alone cost function associated to $c^{*}$, defined as $C^{*}(S, c)=C\left(S, c^{*}\right)$ for all $S \subseteq N$.

Bogomolnaia and Moulin (2010) offer a closed-form expression of the folk solution. Fix $i$ and rearrange the cost $c_{i j}^{*}$ of the $n-1$ edges connecting agent $i$ to other agents in increasing order as $c_{i}^{* k}$ such that $c_{i}^{* 1} \leq c_{i}^{* 2} \leq \ldots \leq c_{i}^{*(n-1)}$. Then, the folk solution $y^{f}(N, c)$ can be written as

$$
y_{i}^{f}(N, c)=\frac{1}{n} c_{0 i}^{*}+\sum_{k=1}^{n-1} \frac{1}{k(k+1)} \min \left\{c_{i}^{* k}, c_{0 i}^{*}\right\} .
$$

Another interpretation, found in Bergantinos and Vidal-Puga (2007b), uses the notion of an optimistic game. This game assigns to any coalition $S$ the cost of connecting its members to the source under the assumption that agents in $N \backslash S$ are already connected and that agents in $S$ can use their locations. We can then define the folk solution as the Shapley value of the corresponding stand-alone game.

By contrast, the Kar solution is the Shapley value of the stand-alone game where a coalition assumes that others are not connected and that it cannot use their locations, also called the pessimistic standalone game.

Even on simple games, the two solutions often propose allocations that are quite different. Consider the following two player problem $\left(\{1,2\}, c^{e_{1}}\right)$.

Example 1 We have $c^{e_{1}}$ and $\left(c^{e_{1}}\right)^{*}$ represented in Figure 1. Agents are identified in the circles and the costs are next to the edges.

\{Insert Figure 1. Caption: Two-agent example\}

We have the following values for $C\left(\cdot, c^{e_{1}}\right)$ and $C\left(\cdot,\left(c^{e_{1}}\right)^{*}\right)$.

$S \quad C\left(S, c^{e_{1}}\right) \quad C\left(S,\left(c^{e_{1}}\right)^{*}\right)$

$\{1\} \quad 0 \quad 0$

$\{2\} \quad 1 \quad 0$

$\{1,2\} \quad 0 \quad 0$

We obtain the following allocations: $y_{1}^{k}\left(\{1,2\}, c^{e_{1}}\right)=-\frac{1}{2}, y_{2}^{k}\left(\{1,2\}, c^{e_{1}}\right)=\frac{1}{2}, y_{1}^{f}\left(\{1,2\}, c^{e_{1}}\right)=0$ and $y_{2}^{f}\left(\{1,2\}, c^{e_{1}}\right)=0$.

\section{Problem Separation and solutions satisfying it}

Before introducing the main new property, we start with a new but very weak property stating that cost shares should not depend on the cost of edges that are never used. An edge $(i, j)$ is irrelevant if $c_{i j}>\max \left\{c_{0 i}, c_{0 j}\right\}$. Such an edge is never used, as it is always preferable to connect agents $i$ and $j$ through the source.

Let $\bar{\Gamma}$ be the set of cost matrices such that there are no irrelevant edges; i.e. $c_{i j} \leq \max \left\{c_{0 i}, c_{0 j}\right\}$ for all $i, j \in N$. Let $\bar{c} \in \bar{\Gamma}$ be the cost matrix with no irrelevant edges associated with $c$. For all $i, j \in N$, $\bar{c}_{i j}=\min \left\{c_{i j}, \max \left\{c_{0 i}, c_{0 j}\right\}\right\}$, while $\bar{c}_{0 i}=c_{0 i}$ for all $i \in N$.

Independence of Irrelevant Edges: For any mcst problem $(N, c), y(N, c)=y(N, \bar{c})$.

Therefore, any solutions satisfying Independence of Irrelevant Edges that coincide on $\bar{\Gamma}$ also coincide on $\Gamma$. This very mild property is satisfied by all usual cost sharing solutions and any solution not satisfying it would depend on edges that have no impact on any costs. It is easy to see that an irrelevant edge will see its cost modified in the irreducible matrix. Therefore, Independence of Irrelevant Edges is implied by the Reductionism property, that imposes a reliance on the irreducible cost matrix. 
Notice that for $c \in \bar{\Gamma}$, there is always an mcst such that only one agent is connected to the source. Therefore, the minimum cost spanning tree problem contains two sub problems: connecting one agent to the source and connecting that agent to all others. We introduce a new property based on this observation that we are able to split a minimum cost spanning tree problem into these two problems. Therefore, applying a cost sharing solution to the whole problem or independently to the sub-problems should yield the same result. Such a property makes sure that if the network is sequentially built in this manner (first selecting who to connect to the source then building the rest of the network) there will be no discussion on doing the cost sharing sequentially or on the whole problem.

Formally, let $\hat{c}$ be the source connection problem associated with $c$ : for all $i \in N, \hat{c}_{0 i}=c_{0 i}$, while $\hat{c}_{i j}=0$ for all $i, j \in N$. Then, all that is left are the costs to connect agents to the source. The mcst is such that one agent is connected to the source $\left(i\right.$ such that $\hat{c}_{0 i} \leq \hat{c}_{0 j}$ for all $\left.j \in N\right)$ and all others are connected to him (at no cost since $\hat{c}_{i j}=0$ for all $i, j \in N$ ).

Let $\tilde{c}$ be the agent connection problem associated with $c$ : for all $i, j \in N, \tilde{c}_{i j}=c_{i j}$, while $\tilde{c}_{0 i}=$ $\max _{e \in N_{0}^{p}} c_{e}$ for all $i \in N$. Then, all agents have the same (high) cost to connect to the source, so the mcst is such that only one (random) agent is connected to the source, and all other agents are connected through this agent. Notice that since we have modified the cost to connect to the source in $\tilde{c}$, we have to subtract that extra cost.

We have this relationship between stand alone costs on $c, \hat{c}$ and $\tilde{c}$ :

Lemma 1 For all mcst problem $(N, c)$ such that $c \in \bar{\Gamma}, C(S, c)=C(S, \hat{c})+C(S, \tilde{c})-\max _{e \in N_{0}^{p}} c_{e}$ for all $S \subseteq N$.

Proof. Since for all $c \in \bar{\Gamma}$ there is always an mcst such that only one agent is connected to the source, this is also true for all $c^{S}$, with $S \subset N$. Then, there is always an mcst for the problem $\left(S, c^{S}\right)$ such that one agent is connected to the source and all other agents are connected to this agent. Let $w^{S}$ be (one of) the cheapest way(s) to connect agents in $S$ using only the edges between agents in $S$ (not using the source). Therefore, $C(S, c)=\min _{i \in S} c_{0 i}+\sum_{e \in w^{S}} c_{e}$.

Consider now the cost matrix $\hat{c}$. Clearly, $C(S, \hat{c})=\min _{i \in S} \hat{c}_{0 i}=\min _{i \in S} c_{0 i}$. Consider the cost matrix $\tilde{c}$. There always is an mcst for the problem $\left(S, \tilde{c}^{S}\right)$ such that one agent is connected to the source and all other agents are connected to this agent. Therefore, $C(S, \tilde{c})=\max _{e \in N_{0}^{p}} c_{e}+\sum_{e \in w^{S}} c_{e}$.

We then have $C(S, \hat{c})+C(S, \tilde{c})-\max _{e \in N_{0}^{p}} c_{e}=\min _{i \in S} c_{0 i}+\max _{e \in N_{0}^{p}} c_{e}+\sum_{e \in w^{S}} c_{e}-\max _{e \in N_{0}^{p}} c_{e}$ which simplifies to $\min _{i \in S} c_{0 i}+\sum_{e \in w^{S}} c_{e}$. Therefore, $C(S, c)=C(S, \hat{c})+C(S, \tilde{c})-\max _{e \in N_{0}^{p}} c_{e}$

Define $\dot{c}$ as follows: $\dot{c}_{0 i}=\max _{e \in N_{0}^{p}} c_{e}$ for all $i \in N$ and $\dot{c}_{e}=0$ otherwise. It is easy to see that $C(S, \dot{c})=\max _{e \in N_{0}^{p}} c_{e}$ for all $S \subseteq N$. Therefore, Lemma 1 can be written as $C(S, c)=C(S, \hat{c})+C(S, \tilde{c})-$ $C(S, \dot{c})$ for all $S \subseteq N .{ }^{1}$ Since we have this relationship between an mcst problem and its associated source connection and agent connection problems, it becomes natural to introduce a property linking the cost shares of the mcst problem to those of the source connection and agent connection problems.

Problem Separation: For any mcst problem $(N, c)$ such that $c \in \bar{\Gamma}(N), y_{i}(N, c)=y_{i}(N, \hat{c})+$ $y_{i}(N, \tilde{c})-y_{i}(N, \dot{c})$ for all $i \in N .^{2}$

This property, however, might be too strong. In fact, the folk solution does not satisfy it. While we have the relationship of Lemma 1, notice that in both the source connection and agent connection problems, we can find the optimal tree by connecting an agent to the source and all others through that agent. While for any $c \in \bar{\Gamma}$ there exists such an optimal tree, we will limit the scope of Problem Separation to a subset of problems where it is completely natural to connect only one agent to the source. This case is as follows: suppose that the smallest cost to connect an agent to the source is $x$. If for any pair of agents $i, j \in N$, we can construct a path $p_{i j}$ in $N$ such that the cost of all edges is no larger than $x$, then clearly the optimal way to go is to connect only one agent to the source.

\footnotetext{
${ }^{1}$ It would be possible to divide a problem $(N, c)$ in its source connection problem and in a minimum cost spanning tree problem without a source, where we need to connect all players together. While this would eliminate the need to define $\dot{c}$, the current format has the advantage that $c, \hat{c}, \tilde{c}$ and $\dot{c}$ are all mcst problems with a source as defined in Section 2 .

${ }^{2}$ If $y$ satisfies Anonymity, then $y_{i}(N, \dot{c})=\frac{1}{|N|} \max _{e \in N_{0}^{P}} c_{e}$ for all $i \in N$. The extra cost we added in the agent connection problem is split evenly among agents.
} 
In many examples associated to mcst problems, it is expected that costs to connect to the source are large compared to costs to connect agents to each other. ${ }^{3}$

Weak Problem Separation: For any mcst problem $(N, c)$ such that $c \in \bar{\Gamma}(N)$, if $c_{e} \leq \min _{i \in N} c_{0 i}$ for all $e \in t^{*}(c)$, all $t^{*} \in T^{*}(c)$, then $y_{i}(N, c)=y_{i}(N, \hat{c})+y_{i}(N, \tilde{c})-y_{i}(N, \dot{c})$ for all $i \in N$.

We therefore restrict the scope of Problem Separation to the set of problems where there is no edge used in an mcst that is more expensive than the cheapest edge connecting an agent to the source. ${ }^{4}$

Weak Problem Separation and Independence of Irrelevant Edges will be used with four other properties, with the next three being already known in the literature and the fourth being a weaker version of another known property.

We use an Anonymity property that says that cost allocated to the agents should not depend on their name. We need the following notation: let $N, N^{\prime} \subset \mathcal{N}$ be such that $|N|=\left|N^{\prime}\right|$ and $f$ be a bijection from $N$ to $N^{\prime}$. For any $c \in \Gamma(N), f c \in \Gamma\left(N^{\prime}\right)$ is such that $(f c)_{f(i) f(j)}=c_{i j}$ and $(f c)_{0 f(k)}=c_{0 k}$ for all $i, j, k \in N$. For any $y \in \mathbb{R}^{N}, f y \in \mathbb{R}^{N^{\prime}}$ is such that $f y_{f(i)}=y_{i}$ for all $i \in N$.

Anonymity: For any mest problem $(N, c)$ and bijection $f$ from $N$ to $N^{\prime}$, we have $f y(N, c)=$ $y\left(N^{\prime}, f c\right)$.

Anonymity is widely used in cooperative game theory (see Moulin (1988)). It implies the usual symmetry property, found in Bergantinos and Vidal-Puga (2009) and Bogomolnaia and Moulin (2010).

We also use one of the properties used to characterize the Kar solution (Kar (2002)), that says that if we can split our agents into two groups that can be connected independently to the source, then we can do the cost sharing separately on these two groups. More precisely, two groups $S$ and $N \backslash S$ can be connected independently to the source if for all $i \in S$ and $j \in N \backslash S, c_{i j} \geq \max \left\{c_{0 i}, c_{0 j}\right\}$. Then, it is always as costly to connect two agents in distinct groups directly one to the other than to connect them both to the source. Notice that a solution that fails Group Independence is unfair, as the cost shares of a group can depend on the situation of a completely independent group of agents.

Group Independence: For any mcst problem $(N, c)$, if $S \subset N$ is such that for all $i \in S$ and $j \in N \backslash S, c_{i j} \geq \max \left\{c_{0 i}, c_{0 j}\right\}$, then $y_{i}(N, c)=\left\{\begin{array}{c}y_{i}\left(S, c^{S}\right) \text { if } i \in S \\ y_{i}\left(N \backslash S, c^{N \backslash S}\right) \text { if } i \in N \backslash S\end{array}\right.$.

Kar (2002) actually uses a weaker version where $S$ and $N \backslash S$ are considered distinct if for all $i \in S$ and $j \in N \backslash S, c_{i j}>\max \left\{c_{0 i}, c_{0 j}\right\}$. Removing the strict inequality adds the case where we are indifferent between connecting agents from distinct groups to each other or independently. The mere fact that the groups can be connected independently seems a sufficient reason to consider the groups as independent.

Note that when we apply Group Independence to a problem $(N, c)$ with $c \in \bar{\Gamma}(N)$, there is no edge $(i, j)$ such that $c_{i j}>\max \left\{c_{0 i}, c_{0 j}\right\}$. Groups $S$ and $N \backslash S$ are considered independent if for all $i \in S$ and $j \in N \backslash S, c_{i j}=\max \left\{c_{0 i}, c_{0 j}\right\}$. Then we are indifferent between connecting agents in $S$ alone or with agents in $N \backslash S$. More importantly, even in this case, there is no gain for coalition $S$ (or any of its subsets) to cooperate with agents in $N \backslash S$ (or any of its subsets), which justifies their cost shares being computed independently.

Next, we define Piecewise Linearity, which says that if we can decompose a cost matrix into submatrices where the cost of all edges are ordered in the same manner as the original matrix, then the cost allocation on the original cost matrix should equal the sum of the cost allocations on the submatrices. This property (or similar versions), a weaker version than the classical Additivity property in the general setting (first proposed by Shapley (1953)), has been used in Bergantinos and Vidal-Puga (2009), Bogomolnaia and Moulin (2010), Branzei et al (2004) and Tijs et al (2006). Piecewise Linearity generates a rich class of solutions having a simple structure. Cost shares can be defined on simple elementary matrices where costs of all edges are either 0 or 1 , making it particularly appealing. In

\footnotetext{
${ }^{3}$ In their study of Monotonicity and Ranking properties, Bogomolnaia and Moulin (2010) use a stronger but similar restriction. They apply their properties to the set of cost matrices such that for all $i, j \in N, c_{i j} \leq \min _{k \in N} c_{0 k}$. That is, they suppose that all connection costs between agents are smaller than all connection costs to the source.

${ }^{4}$ Weak Problem Separation is implied by Restricted Addivity, a property used in Bergantinos and Vidal-Puga (2009), among others. See Lemma A.1 for details. Thanks to an anonymous referee for pointing this out.
} 
addition, many normative properties easily defined on those elementary matrices automatically extend to arbitrary matrices.

To formally define the Piecewise Linearity property we need the following notation. Suppose $N=\{1, \ldots, n\}$ and denote arbitrarily the $q=\frac{n(n+1)}{2}$ distinct edges in $N_{0}^{p}$, such that $c=\left(c_{e^{1}}, \ldots, c_{e^{q}}\right)$. For any permutation $\sigma$ of $\{1, \ldots, q\}$, define $K_{\sigma}(N)=\left\{c \in \Gamma(N) \mid c_{e^{\sigma(1)}} \leq c_{e^{\sigma(2)}} \leq \ldots \leq c_{e^{\sigma(q)}}\right\}$ to be the cone in $\Gamma(N)$ containing all cost matrices with a given increasing ordering of connection costs. Note that $\Gamma(N)=\cup K_{\sigma}(N)$. A cost sharing solution $y$ is piecewise linear if it is linear in $K_{\sigma}$ for all $\sigma$. More precisely, denote by $\Gamma^{e}$ the set of elementary cost matrices where all connection costs are either 0 or $1: \Gamma^{e}(N)=\left\{c \in \Gamma(N): c_{e} \in\{0,1\}\right.$ for all $\left.e \in N_{0}^{p}\right\}$. For any cone $K_{\sigma}(N)$ and any $k \in\{1, \ldots, q\}$, let $b^{k} \in \Gamma^{e}(N)$ be such that $b_{e^{\sigma(1)}}^{k}=\ldots=b_{e^{\sigma(k-1)}}^{k}=0$ while $b_{e^{\sigma(k)}}^{k}=\ldots=b_{e^{\sigma(q)}}^{k}=1$.

Piecewise Linearity: For any mcst problem $(N, c), y(N, c)=\sum_{k=1}^{q}\left(c_{e^{\sigma(k)}}-c_{e^{\sigma(k-1)}}\right) y\left(N, b^{k}\right)$ for any $\sigma$ and any $c \in K_{\sigma}(N)$.

Therefore, if solutions satisfying Piecewise Linearity coincide on $\Gamma^{e}$, they also coincide on $\Gamma$. Let $y$ be a solution defined over $\Gamma^{e}$. The piecewise linear extension of $y$ is a solution $y^{L}$ such that for all $c \in K_{\sigma}(N), y^{L}(N, c)=\sum_{k=1}^{q}\left(c_{e^{\sigma(k)}}-c_{e^{\sigma(k-1)}}\right) y\left(N, b^{k}\right)$.

Having defined the set of elementary matrices, we define the subsets of elementary matrices that correspond to source connection and agent connection problems. The set of elementary cost matrices generating source connection problems is $\hat{\Gamma}^{e}$, the set of elementary matrices $c$ such that $c_{i j}=0$ for all $i, j \in N$. The set of elementary cost matrices generating agent connection problems is $\tilde{\Gamma}^{e}$, the set of elementary matrices $c$ such that $c_{0 i}=1$ for all $i \in N$.

Finally, we use a weak version of the Equal Treatment property used in Kar (2002), that says that if the cost of edge $(i, j)$ changes, with all other costs staying put, then the cost shares of $i$ and $j$ should be changed by the same amount. This introduces the concept of equal responsibility of $i$ and $j$ over the edge between them. While it is used to characterize the Kar solution, it is not satisfied by the folk solution, as a change in the cost of edge $(i, j)$ might change the irreducible cost matrix in a way that impacts $i$ and $j$ differently. We use a weaker version that is restricted to edges not used in any mcst.

Weak Equal Treatment: For any mcst problems $(N, c)$ and $\left(N, c^{\prime}\right)$ and $i, j \in N$ such that $c_{i j}>c_{i j}^{\prime}, c_{e}=c_{e}^{\prime}$ else and $C(N, c)=C\left(N, c^{\prime}\right)$, we have $y_{i}(N, c)-y_{i}\left(N, c^{\prime}\right)=y_{j}(N, c)-y_{j}\left(N, c^{\prime}\right)$.

While the original version is very appealing from a normative point of view, the weak version at least makes sure that the principle is respected for edges not in any mcst. Note that it is implied by the Reductionist property, used to characterize the folk solution, that states that the cost shares should not be responsive to costs of edges not in any mcst.

Before moving on to the main theorem, we define some additional notations. Let $R(c) \subseteq N$ be the set of agents such that $c_{0 i}=1$. Any $c \in \hat{\Gamma}^{e}(N)$ is uniquely defined by $R(c)$. Let $Z^{i}(c)=$ $\left\{j \in N \backslash\{i\} \mid c_{i j}=0\right\}$ be the set of agents to which $i$ has a free connection. Any $c \in \tilde{\Gamma}^{e}(N)$ is uniquely defined by $Z^{1}, Z^{2}, \ldots, Z^{|N|}$. Let $N_{i}(c)=\left\{j \in N \mid\right.$ there exists a free path $p_{i j}$ in $\left.N\right\}$. Of course, $Z^{i}(c) \subseteq$ $N_{i}(c)$. Also, since $i \in N_{i}(c)$, we always have that $N_{i}(c) \neq \emptyset$. Let $\tilde{\Gamma}_{N C}^{e}(N)$ be the set of elementary matrices $c$ with $c_{0 i}=1$ and such that there are no free cycles $p_{i i}$ in $N$, for all $i \in N$.

The following theorem shows that there is a family of solutions satisfying the set of properties defined above. These solutions are the affine combination of the Kar and folk solutions. We write the weights such that they depend on $a$, the cost share of agent 2 in Example 1. Therefore, by determining what we deem fair as a solution in Example 1, we can determine the weight to put on the Kar and folk solutions. The properties in the theorem are independent, as shown in Lemma A.2, in appendix.

Theorem 1 Let $y^{a}=2 a\left(y^{k}-y^{f}\right)+y^{f}$ and $Y=\left\{y^{a} \mid a \in \mathbb{R}\right\}$. A solution y satisfies Weak Problem Separation, Group Independence, Piecewise Linearity, Anonymity, Weak Equal Treatment and Independence of Irrelevant Edges if and only if $y \in Y$. In addition, $a=y_{2}\left(\{1,2\}, c^{e_{1}}\right)$, the cost share of agent 2 in Example 1.

Proof. Lemma A.1, in the appendix, shows that all solutions in $Y$ satisfy the six properties. Let $y$ be a solution that satisfies Weak Problem Separation, Group Independence, Piecewise Linearity, 
Anonymity, Weak Equal Treatment and Independence of Irrelevant Edges. Let $a=y_{2}\left(\{1,2\}, c^{e_{1}}\right)$ and $y^{a}=2 a\left(y^{k}-y^{f}\right)+y^{f}$. We show that $y=y^{a}$.

Part 1: $y(N, c)=y^{a}(N, c)$ for all $c \in \hat{\Gamma}^{e}$, the set of problems that generate elementary source connection problems

We start by computing $y$. To do so, we first compute cost shares as functions of $N$ and $R(c)$. Next, we define two problems that generate the same agent connection problem and that can be separated in two source connection problems using Group Independence. The equations that we obtain allow us to conclude that the cost shares depend only on $|N|$, and that there is a well defined relationship between different levels of $|N|$.

For $c \in \hat{\Gamma}^{e}(N)$, if $R(c)=N$, then $C(N, c)=1$. By Anonymity and budget balance, $y_{i}(N, c)=\frac{1}{|N|}$.

Let $R \subsetneq N$ and $c^{N, R} \in \Gamma^{e}$ be such that $c_{0 i}^{N, R}=\left\{\begin{array}{c}1 \text { if } i \in R \\ 0 \text { if } i \in N \backslash R\end{array}\right.$. Then, we define $a_{|R|}^{|N|} \equiv y_{i}\left(N, \hat{c}^{N, R}\right)$ for all $i \in R$. By budget balance and Anonymity, we have $y_{i}\left(N, \hat{c}^{N, R}\right)=-\frac{|R| a_{|R|}^{|N|}}{|N|-|R|}$ for $i \in N \backslash R$.

Let $n=|N|$. Fix $i \in N$ and let $c \in \Gamma^{e}, S \subset N \backslash\{i\}$ with $|S|=m$ be such that we have the following three conditions i) for all $j, k \in N \backslash\{i\}, c_{j k}=0$ ii) $c_{i j}=0$ if $j \in S$ and $c_{i j}=1$ else and iii) $c_{0 j}=0$ for all $j \in N \backslash\{i\}, c_{0 i}=1$.

By Weak Problem Separation and Anonymity, $y_{l}(N, c)=y_{l}(N, \hat{c})+y_{l}(N, \tilde{c})-\frac{1}{n}$ for all $l \in N$, with $y(N, \hat{c})$ such that $y_{i}(N, \hat{c})=a_{1}^{n}$ and $y_{j}(N, \hat{c})=-\frac{a_{1}^{n}}{n-1}$ for all $j \in N \backslash\{i\}$.

Notice that for $k \in S \cup\{i\}$ and $l \in N \backslash(S \cup\{i\}), c_{k l} \geq \max \left\{c_{0 k}, c_{0 l}\right\}$. Therefore, by Group Independence, $y_{k}(N, c)=y_{k}\left(S \cup\{i\}, c^{S \cup\{i\}}\right)$ for all $k \in S \cup\{i\}$ and $y_{l}(N, c)=y_{l}\left(N \backslash(S \cup\{i\}), c^{N \backslash(S \cup\{i\})}\right)$ for all $l \in N \backslash(S \cup\{i\})$.

The problem $\left(S \cup\{i\}, c^{S \cup\{i\}}\right)$ is such that $c_{k l}^{S \cup\{i\}}=0$ for all $k, l \in S \cup\{i\}, c_{0 j}^{S \cup\{i\}}=0$ for $j \in S$, and $c_{0 i}^{S \cup\{i\}}=1$. Therefore, $y_{i}\left(S \cup\{i\}, c^{S \cup\{i\}}\right)=a_{1}^{m+1}$.

The problem $\left(N \backslash(S \cup\{i\}), c^{N \backslash(S \cup\{i\})}\right)$ is such that $c_{k l}^{N \backslash(S \cup\{i\})}=0$ for all $k, l \in N_{0} \backslash(S \cup\{i\})$. Therefore, by Anonymity, $y_{k}\left(N \backslash(S \cup\{i\}), c^{N \backslash(S \cup\{i\})}\right)=0$ for all $k \in N \backslash(S \cup\{i\})$.

Combining these results, we obtain

$$
\begin{aligned}
& y_{i}(N, \tilde{c})=\frac{1}{n}+a_{1}^{m+1}-a_{1}^{n} \\
& y_{j}(N, \tilde{c})=\frac{1}{n}+\frac{a_{1}^{n}}{n-1} \text { for } j \in N \backslash(S \cup\{i\}) .
\end{aligned}
$$

Consider $c^{\prime}$ such that $c_{k l}^{\prime}=c_{k l}$ if $k, l \neq 0, c_{0 i}^{\prime}=0, c_{0 j}^{\prime}=0$ if $j \in S$ and $c_{0 j}^{\prime}=1$ if $j \in N \backslash(S \cup\{i\})$.

By Weak Problem Separation and Anonymity, $y_{l}\left(N, c^{\prime}\right)=y_{l}\left(N, \hat{c}^{\prime}\right)+y_{l}\left(N, \tilde{c}^{\prime}\right)-\frac{1}{n}$ for all $l \in N$, with $y\left(N, \hat{c}^{\prime}\right)$ such that $y_{j}\left(N, \hat{c}^{\prime}\right)=a_{n-m-1}^{n}$ if $j \in N \backslash(S \cup\{i\})$ and $y_{j}\left(N, \hat{c}^{\prime}\right)=-\frac{(n-m-1) a_{n-m-1}^{n}}{m+1}$ for all $j \in S \cup\{i\}$. Also, notice that $\tilde{c}^{\prime}=\tilde{c}$, so $y\left(N, \tilde{c}^{\prime}\right)=y(N, \tilde{c})$.

Notice also that for $j \in N \backslash\{i\}, c_{i j}^{\prime} \geq \max \left\{c_{0 i}^{\prime}, c_{0 j}^{\prime}\right\}$. Therefore, by Group Independence, $y_{j}\left(N, c^{\prime}\right)=$ $y_{j}\left(N \backslash\{i\}, c^{\prime N \backslash\{i\}}\right)$ for all $j \in N \backslash\{i\}$ and $y_{i}\left(N, c^{\prime}\right)=y_{i}\left(\{i\}, c^{\prime\{i\}}\right)$.

The problem $\left(N \backslash\{i\}, c^{\prime N \backslash\{i\}}\right)$ is such that $c_{k l}^{\prime N \backslash\{i\}}=0$ for all $k, l \in N \backslash\{i\}, c_{0 j}^{\prime N \backslash\{i\}}=0$ for $j \in S$, and $c_{0 j}^{\prime N \backslash\{i\}}=1$ for $j \in N \backslash(S \cup\{i\})$. Therefore, $y_{j}\left(N \backslash\{i\}, c^{\prime N \backslash\{i\}}\right)=a_{n-m-1}^{n-1}$ for all $j \in N \backslash(S \cup\{i\})$.

By budget balance, $y_{i}\left(\{i\}, c^{\prime\{i\}}\right)=c_{0 i}^{\prime}=0$.

Putting these results together with the values of $y(N, \tilde{c})$ found above, we obtain

$$
\begin{aligned}
& y_{i}\left(N, c^{\prime}\right)=-\frac{(n-m-1) a_{n-m-1}^{n}}{m+1}+a_{1}^{m+1}-a_{1}^{n}=0 \\
& y_{j}\left(N, c^{\prime}\right)=a_{n-m-1}^{n}+\frac{a_{1}^{n}}{n-1}=a_{n-m-1}^{n-1} \text { for } j \in N \backslash(S \cup\{i\}) .
\end{aligned}
$$


This gives us the following equations:

$$
\begin{aligned}
-\frac{(n-m-1) a_{n-m-1}^{n}}{m+1}+a_{1}^{m+1}-a_{1}^{n} & =0 \\
a_{n-m-1}^{n}+\frac{a_{1}^{n}}{n-1}-a_{n-m-1}^{n-1} & =0 .
\end{aligned}
$$

Notice that by our assumptions $1 \leq m \leq n-2$.

In (2), let $m=n-2$. We obtain

$$
\frac{n}{n-1} a_{1}^{n}=a_{1}^{n-1}
$$

By iteration, we obtain

$$
a_{1}^{k}=\frac{n}{k} a_{1}^{n} \text { for } 2 \leq k \leq n .
$$

Replacing this in (1), we obtain

$$
\frac{n-m-1}{m+1} a_{n-m-1}^{n}+a_{1}^{n}=a_{1}^{m+1}=\frac{n}{m+1} a_{1}^{n}
$$

which simplifies to $a_{n-m-1}^{n}=a_{1}^{n}$ for $1 \leq m \leq n-2$, which can be restated as $a_{1}^{n}=a_{k}^{n} \equiv a^{n}$ for $2 \leq k \leq n-2$. It remains to show that $a_{n-1}^{n}=a^{n}$.

In (2), let $m=1$. We obtain

$$
\frac{n}{n-1} a_{1}^{n}=a_{n-2}^{n-1} \text {. }
$$

Therefore, by (3) and the result above, $a_{n-2}^{n-1}=a_{1}^{n-1}=a^{n-1}$. These results hold for any values of $n$, so we can conclude that for all $k \geq 2,1 \leq l \leq k-1, a_{l}^{k}=a^{k}$. Then, by iteration on (3), we get $a^{k}=\frac{2 a^{2}}{k}$.

Therefore, for $c \in \hat{\Gamma}^{e}$, if $R(c)=N, y_{i}(N, c)=\frac{1}{|N|}$ and if $R(c) \neq N, y_{i}(N, c)=\left\{\begin{array}{c}\frac{2 a^{2}}{|N|} \text { if } i \in R(c) \\ -\frac{|R(c)| 2 a^{2}}{(|N|-|R(c)|)|N|} \text { if } i \in N \backslash R(c)\end{array}\right.$, with $a^{2} \in \mathbb{R}$.

Next, we compute $y^{a}(N, c)$ for $c \in \hat{\Gamma}^{e}$. We can check that if $R(c)=N, y_{i}^{k}(N, c)=y_{i}^{f}(N, c)=\frac{1}{|N|}$ and if $R(c) \neq N, y_{i}^{k}(N, c)=\left\{\begin{array}{c}\frac{1}{|N|} \text { if } i \in R(c) \\ -\frac{|R(c)|}{(|N|-|R(c)|)|N|} \text { if } i \in N \backslash R(c)\end{array}\right.$ and $y_{i}^{f}(N, c)=0$ for all $i \in N .{ }^{5}$ Therefore, for $c \in \hat{\Gamma}^{e}$, if $R(c)=N, y_{i}^{a}(N, c)=\frac{1}{|N|}$. If $R(c) \neq N, y_{i}^{a}(N, c)=\left\{\begin{array}{c}\frac{2 a}{|N|} i \in R(c) \text { and } R(c) \neq N \\ -\frac{|R(c)| 2 a}{(|N|-|R(c)|)|N|} \text { if } i \in N \backslash R(c)\end{array}\right.$. Notice that $c^{e_{1}}=\hat{c}^{\{1,2\},\{2\}}$. Therefore, $a=y_{2}\left(\{1,2\}, c^{e_{1}}\right)=a^{2}$ and $y(N, c)=y^{a}(N, c)$ for all $c \in \hat{\Gamma}^{e}$.

Part 2: $y(N, \tilde{c})=y^{a}(N, \tilde{c})$ for all $\tilde{c} \in \tilde{\Gamma}_{P N C}^{e}$, the set of elementary matrices generating agent connection problems and such that the free edges constitute a tree connecting all agents.

First, we show that for $\tilde{c} \in \tilde{\Gamma}_{P N C}^{e}, y_{i}(N, \tilde{c})=a\left(2-\left|Z^{i}(\tilde{c})\right|\right)-\frac{(2 a-1)}{\left|N_{i}(\tilde{c})\right|}$.

For all $\tilde{c} \in \tilde{\Gamma}_{P N C}^{e}$, there is a unique free path $p_{i j}^{f}(\tilde{c})$ between any agents $i$ and $j$. For $\tilde{c} \in \tilde{\Gamma}_{P N C}^{e}$, $i, j \in N$, let $N_{j}^{-i}(\tilde{c})=\left\{k \in N \mid p_{j k}^{f}(\tilde{c})\right.$ is in $\left.N \backslash\{i\}\right\} \cdot N_{j}^{-i}$ represents the agents to which $j$ can connect to freely without $i$. Then, for $S \subseteq N \backslash\{i\}, D_{i}^{S}(\tilde{c}) \equiv \cup_{j \in S} N_{j}^{-i}(\tilde{c})$ represents the players in $S$ plus the agents $k$ for which there is a free path $p_{j k}$ in $N \backslash\{i\}$ connecting him to an agent $j \in S$. It represents the set of agents to which agent $i$ can connect to freely with the help of agents in $S$. By definition, $S \subseteq D_{i}^{S}(\tilde{c})$. Since $Z_{i}(\tilde{c})$ is the set of players for which the direct connection to $i$ is free, we have that $D_{i}^{Z_{i}(\tilde{c})}(\tilde{c})=N \backslash\{i\}$. Let $d_{i}^{S}(\tilde{c})=\left|D_{i}^{S}(\tilde{c})\right|$.

\footnotetext{
${ }^{5}$ If $i \in R(c)$, the incremental cost of agent $i$ is 1 when he joins the empty set, and 0 otherwise. Therefore, $y_{i}^{k}=\frac{1}{|N|}$. If $i \notin R(c)$, his incremental cost is -1 if he joins a group of players that are all in $R(c)$ and 0 otherwise. If $R(c) \neq N$, then the irreducible matrix is such that $c_{e}^{*}=0$ for all $e$ and thus $y_{i}^{f}=0$. If $R(c)=N$, then $c^{*}=c$ and $y_{i}^{f}=\frac{1}{|N|}$.
} 
In the following, we consider $c$, and $c^{\prime}$ such that $\tilde{c}=\tilde{c}^{\prime}$. In order to simplify, we write $D_{i}^{S}$ and $d_{i}^{S}$ instead of $D_{i}^{S}(\tilde{c})$ and $d_{i}^{S}(\tilde{c})$.

To make this notation clear, consider the example in Figure 2. It is such that $N=\{1,2,3,4,5,6,7\}$ and the free edges are represented in the figure. Since everyone is connected and there are no free cycles, then the cost matrix is in $\tilde{\Gamma}_{P N C}^{e}$. Consider agent 1 . We have $Z^{1}=\{2,3,4\}$ and $N_{2}^{-1}=\{2\}$, $N_{3}^{-1}=N_{5}^{-1}=\{3,5\}, N_{4}^{-1}=N_{6}^{-1}=N_{7}^{-1}=\{4,6,7\}$. Therefore, we have, among others, $D_{1}^{\{2,3\}}=$ $\{2,3,5\}, D_{1}^{\{3,4\}}=\{3,4,5,6,7\}$ and $D_{1}^{\{2,3,4\}}=N \backslash\{1\}$.

$\left\{\right.$ Insert Figure 2. Caption: Example of cost matrix in $\left.\tilde{\Gamma}_{P N C}^{e} \cdot\right\}$

The proof consists in "cutting off" the paths connected to $i$ one by one, until $i$ is left alone, and using Group Independence and Weak Problem Separation to find cost shares. In the above example, we start first by letting $c_{01}=c_{02}=0$, with $c_{0 k}=1$ otherwise. Using Weak Problem Separation and the results of Part 1 , we can express $y_{1}(N, c)$ as a function of $y_{1}(N, \tilde{c})$. We can also

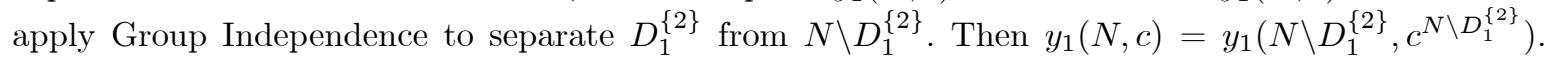
Applying Weak Problem Separation and the results of Part 1 again, we obtain $y_{1}(N, c)$ as a function

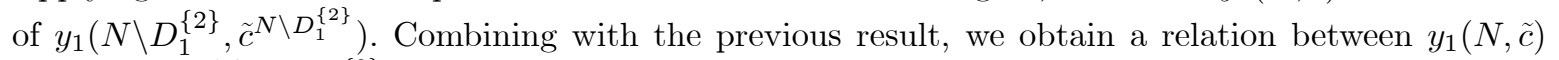

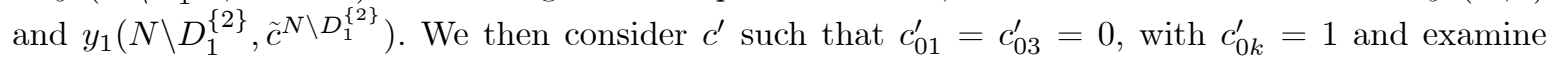

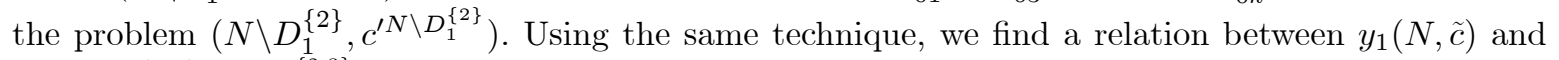
$y_{1}\left(N \backslash D_{1}^{\{2,3\}}, \tilde{c}^{\left.N \backslash D_{1}^{\{2,3\}}\right)}\right.$. Doing the same thing with agent 4 allows us to find a relation between $y_{1}(N, \tilde{c})$

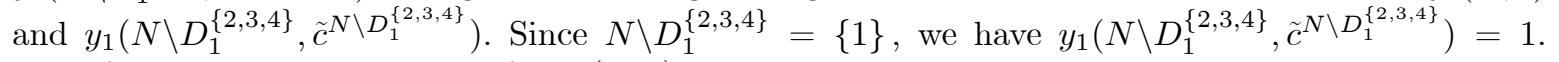
Therefore, we can directly solve for $y_{1}(N, \tilde{c})$.

We now return to the general case. Suppose that $\left|Z^{i}(\tilde{c})\right|=m$ and that $|N|=n$. Take any ordering of the agents in $Z^{i}(\tilde{c}), j_{1}, j_{2}, \ldots, j_{m}$. The proof contains $m$ steps, one for each agent in $Z^{i}(\tilde{c})$.

Step 1: Define $c$ such that $c_{j k}=\tilde{c}_{j k}$ for all $j, k \in N, c_{0 i}=c_{0 j_{1}}=0$ and $c_{0 k}=1$ else. By Weak Problem Separation and Anonymity, we have that $y_{i}(N, c)=-\frac{a(n-2)}{n}+y_{i}(N, \tilde{c})-\frac{1}{n}$. By definition of $\tilde{\Gamma}_{P N C}^{e}$, for any $j, k \in N$, there is a unique free path from $j$ to $k$ in $N$. For $j \in N \backslash D_{i}^{\left\{j_{1}\right\}}$ and $k \in D_{i}^{\left\{j_{1}\right\}}$, this path has to go through $i$ and $j_{1}$ and thus $c_{j k} \geq \max \left\{c_{0 j}, c_{0 k}\right\}$ and we can apply Group Independence. We have that $y_{i}(N, c)=y_{i}\left(N \backslash D_{i}^{\left\{j_{1}\right\}}, c^{N \backslash D_{i}^{\left\{j_{1}\right\}}}\right)$. By Weak Problem Separation and

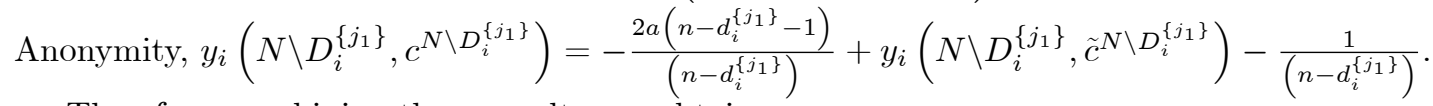

Therefore, combining these results, we obtain

$$
y_{i}(N, \tilde{c})=y_{i}\left(N \backslash D_{i}^{\left\{j_{1}\right\}}, \tilde{c}^{N \backslash D_{i}^{\left\{j_{1}\right\}}}\right)+\frac{a(n-2)}{n}-\frac{2 a\left(n-d_{i}^{\left\{j_{1}\right\}}-1\right)}{\left(n-d_{i}^{\left\{j_{1}\right\}}\right)}+\frac{1}{n}-\frac{1}{\left(n-d_{i}^{\left\{j_{1}\right\}}\right)} .
$$

Step $l$, for $l=2, \ldots, m-1$. Define $c^{\prime}$ such that $c_{k l}^{\prime}=c_{k l}$ for $k, l \in N, c_{0 i}^{\prime}=c_{0 j_{l}}^{\prime}=0, c_{0 k}^{\prime}=1$ else. By

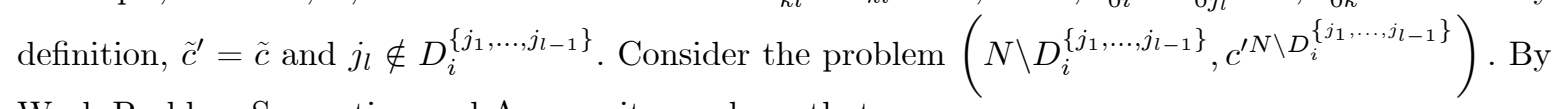
Weak Problem Separation and Anonymity, we have that

$$
\begin{aligned}
y_{i}\left(N \backslash D_{i}^{\left\{j_{1}, \ldots, j_{l-1}\right\}}, c^{\left.\prime N \backslash D_{i}^{\left\{j_{1}, \ldots, j_{l-1}\right\}}\right)=}\right. & -\frac{a\left(n-d_{i}^{\left\{j_{1}, \ldots, j_{l-1}\right\}}-2\right)}{\left(n-d_{i}^{\left\{j_{1}, \ldots, j_{l-1}\right\}}\right)}+y_{i}\left(N \backslash D_{i}^{\left\{j_{1}, \ldots, j_{l-1}\right\}}, \tilde{c}^{\left.N \backslash D_{i}^{\left\{j_{1}, \ldots, j_{l-1}\right\}}\right)}\right. \\
& -\frac{1}{\left(n-d_{i}^{\left\{j_{1}, \ldots, j_{l-1}\right\}}\right)} .
\end{aligned}
$$

By definition of $\tilde{\Gamma}_{P N C}^{e}$, for any $j, k \in N$, there is a unique free path from $j$ to $k$ in $N \backslash D_{i}^{\left\{j_{1}, \ldots, j_{l-1}\right\}}$. For $j \in N \backslash\left(D_{i}^{\left\{j_{1}, \ldots, j_{l-1}\right\}} \cup D_{i}^{\left\{j_{l}\right\}}\right)$ and $k \in D_{i}^{\left\{j_{l}\right\}}$, this path has to go through $i$ and $j_{l}$ and thus $c_{j k} \geq$ 


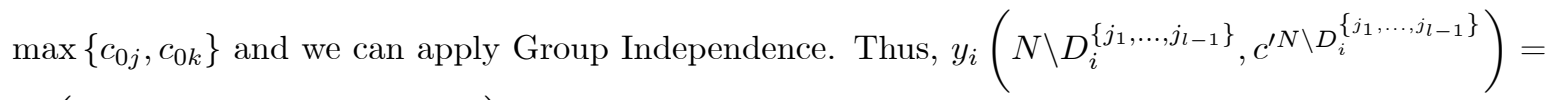

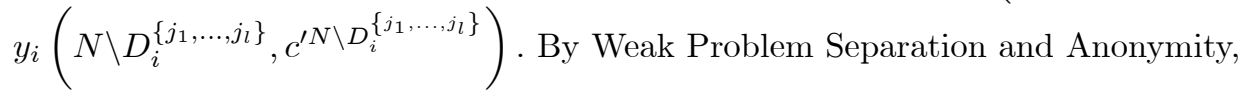

$$
\begin{aligned}
& y_{i}\left(N \backslash D_{i}^{\left\{j_{1}, \ldots, j_{l}\right\}}, c^{\prime N \backslash D_{i}^{\left\{j_{1}, \ldots, j_{l}\right\}}}\right)=-\frac{2 a\left(n-d_{i}^{\left\{j_{1}, \ldots, j_{l}\right\}}-1\right)}{\left(n-d_{i}^{\left\{j_{1}, \ldots, j_{l}\right\}}\right)}+y_{i}\left(N \backslash D_{i}^{\left\{j_{1}, \ldots, j_{l}\right\}}, \tilde{c}^{\left.N \backslash D_{i}^{\left\{j_{1}, \ldots, j_{l}\right\}}\right)}\right. \\
& -\frac{1}{\left(n-d_{i}^{\left\{j_{1}, \ldots, j_{l}\right\}}\right)} \text {. }
\end{aligned}
$$

Combining these results, we obtain

$$
\begin{aligned}
& y_{i}\left(N \backslash D_{i}^{\left\{j_{1}, \ldots, j_{l-1}\right\}}, \tilde{c}^{N \backslash D_{i}^{\left\{j_{1}, \ldots, j_{l-1}\right\}}}\right)=y_{i}\left(N \backslash D_{i}^{\left\{j_{1}, \ldots, j_{l}\right\}}, \tilde{c}^{N \backslash D_{i}^{\left\{j_{1}, \ldots, j_{l}\right\}}}\right)+\frac{a\left(n-d_{i}^{\left\{j_{1}, \ldots, j_{l-1}\right\}}-2\right)}{\left(n-d_{i}^{\left\{j_{1}, \ldots, j_{l-1}\right\}}\right)} \\
& -\frac{2 a\left(n-d_{i}^{\left\{j_{1}, \ldots, j_{l}\right\}}-1\right)}{\left(n-d_{i}^{\left\{j_{1}, \ldots, j_{l}\right\}}\right)}+\frac{1}{\left(n-d_{i}^{\left\{j_{1}, \ldots, j_{l-1}\right\}}\right)}-\frac{1}{\left(n-d_{i}^{\left\{j_{1}, \ldots, j_{l}\right\}}\right)}
\end{aligned}
$$

and

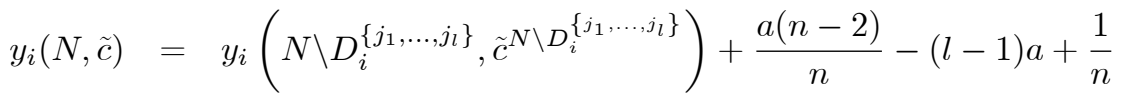

$$
\begin{aligned}
& -\frac{1}{\left(n-d_{i}^{\left\{j_{1}, \ldots, j_{l}\right\}}\right)}-\frac{2 a\left(n-d_{i}^{\left\{j_{1}, \ldots, j_{l}\right\}}-1\right)}{\left(n-d_{i}^{\left\{j_{1}, \ldots, j_{l}\right\}}\right)} .
\end{aligned}
$$

Step $m$. By definition, $N \backslash D_{i}^{\left\{j_{1}, \ldots, j_{m}\right\}}=\{i\}$ and $\tilde{c}^{N \backslash D_{i}^{\left\{j_{1}, \ldots, j_{m}\right\}}}$ is such that $c_{0 i}=1$. Therefore, $y_{i}\left(\{i\}, \tilde{c}^{N \backslash D_{i}^{\left\{j_{1}, \ldots, j_{m}\right\}}}\right)=1$. We also have that $n-d_{i}^{\left\{j_{1}, \ldots, j_{m}\right\}}=1$. Therefore, we have that for all $\tilde{c} \in \tilde{\Gamma}_{P N C}^{e}$

$$
\begin{aligned}
y_{i}(N, \tilde{c}) & =\frac{a(n-2)}{n}-(m-1) a+\frac{1}{n} \\
& =a\left(2-\left|Z^{i}\right|\right)-\frac{(2 a-1)}{|N|} .
\end{aligned}
$$

Next, we compute $y^{a}(N, c)$ for $\tilde{c} \in \tilde{\Gamma}_{P N C}^{e}$. Consider $\tilde{c} \in \tilde{\Gamma}_{P N C}^{e}$. We can check that $y_{i}^{k}(N, \tilde{c})=$ $1-\frac{\left|Z^{i}(\tilde{c})\right|}{2}$ and $y_{i}^{f}(N, \tilde{c})=\frac{1}{|N|} \cdot{ }^{6}$ Therefore, for $c \in \tilde{\Gamma}_{P N C}^{e}, y_{i}^{a}(N, c)=a\left(2-\left|Z^{i}(c)\right|\right)-\frac{(2 a-1)}{|N(c)|}=y_{i}(N, c)$.

Part 3: $y(N, \tilde{c})=y^{a}(N, \tilde{c})$ for all $\tilde{c} \in \tilde{\Gamma}_{P^{*}}^{e}$, the set of elementary matrices generating agent connection problems and such that there exists a free cycle that includes all agents in $N$.

The result of Part 3 is obtained by showing that if two cost sharing solutions coincide for $c \in \tilde{\Gamma}_{P^{*}}^{e}(N)$ when it contains $k$ free edges, they also coincide when it contains $k+1$ free edges. This part of the proof uses Weak Equal Treatment and is very similar to the proof of the main theorem in Kar (2002).

\footnotetext{
${ }^{6}$ Agent $i$ has a marginal contribution of 1 if he joins a coalition that does not include any member of $Z^{i}$. The probability of such an event is $\frac{1}{\left|Z^{i}\right|+1}$. His marginal contribution is $-(k-1)$ when he joins a coalition that contains $k$ agents in $Z^{i}$ (as these agents cannot have a free link between them, as we have no cycles). Thus, $y_{i}^{k}=\frac{1}{\left|Z^{i}\right|+1}-\sum_{k=2}^{\left|Z^{i}\right|} \frac{\left|Z^{i}\right|(k-1)}{\left(\left|Z^{i}\right|-k\right) ! k !}\left(\sum_{l=0}^{|N|-\left|Z^{i}\right|-1} \frac{\left(|N|-\left|Z^{i}\right|-1\right) !}{l !\left(|N|-\left|Z^{i}\right|-1-l\right) !} \frac{(l+k) !(|N|-k-l-1) !}{|N| !}\right)$, which simplifies to $1-\frac{\left|Z^{i}\right|}{2}$. As for the folk solution, the irreducible matrix is $\tilde{c}_{j k}^{*}=0$ for any $j, k \in N$. Therefore, $y_{i}^{f}=\frac{1}{|N|}$.
} 
Suppose that $\bar{y}(N, c)$ and $z(N, c)$ are cost sharing solutions that satisfy all properties and that coincide for $c \in \tilde{\Gamma}_{P^{*}}^{e}(N)$ containing $k-1$ free edges. We show that they coincide if $c$ contains $k$ free edges.

Let $c^{-e}$ be such that $c_{e}^{-e}=1$ and $c_{e^{\prime}}^{-e}=c_{e^{\prime}}$ if $e^{\prime} \neq e$. Let $W=\left\{e \in N^{p} \mid c_{e}=0\right\}$.

Suppose that $(i, j) \in W$. We have

$$
\begin{aligned}
\bar{y}_{i}(N, c)-\bar{y}_{j}(N, c) & =\bar{y}_{i}\left(N, c^{-i j}\right)-\bar{y}_{j}\left(N, c^{-i j}\right) \text { (By Weak Equal Treatment) } \\
& =z_{i}\left(N, c^{-i j}\right)-z_{j}\left(N, c^{-i j}\right) \text { (By induction argument) } \\
& =z_{i}(N, c)-z_{j}(N, c) \text { (By Weak Equal Treatment) }
\end{aligned}
$$

Therefore, we have $\bar{y}_{i}(N, c)-z_{i}(N, c)=\bar{y}_{j}(N, c)-z_{j}(N, c)$ for all $(i, j) \in W$. Let $f=\left\{\left(i_{1}, i_{2}\right), \ldots,\left(i_{K}, i_{K+1}\right)\right\}$ such that $i_{1}=i_{K+1}$ be a free cycle that includes all players in $N$. By definition, if $e \in f$, then $e \in W$. Applying sequentially the fact that $\bar{y}_{i_{k}}(N, c)-z_{i_{k}}(N, c)=\bar{y}_{i_{k+1}}(N, c)-z_{i_{k+1}}(N, c)$ for all $\left(i_{k}, i_{k+1}\right) \in f$, we obtain that

$$
\bar{y}_{i}(N, c)-z_{i}(N, c)=\bar{y}_{j}(N, c)-z_{j}(N, c) \text { for all } i, j \in N .
$$

By efficiency, we have that $\sum_{i \in N}\left(\bar{y}_{i}(N, c)-z_{i}(N, c)\right)=0$. With (4), it implies that

$$
\bar{y}_{i}(N, c)=z_{i}(N, c) \text { for all } i \in N
$$

Therefore, $\bar{y}(N, c)=z(N, c)$.

To conclude the argument, consider the case where $c \in \tilde{\Gamma}_{P^{*}}^{e}(N)$ contains exactly $|N|$ free edges, meaning that there exists an ordering of the agents $i_{1}, \ldots, i_{|N|}$ such that $c_{i_{k} i_{k+1}}=0$ for all $k=$ $1, \ldots,|N|-1, c_{i_{1} i_{|N|}}=0$ and $c_{e}=1$ otherwise. Clearly, for any $e \in W, c^{-e} \in \tilde{\Gamma}_{P N C}^{e}$ and by part 2, all solutions satisfying the properties coincide for $c^{-e}$. Therefore they also coincide for $c \in \tilde{\Gamma}_{P^{*}}^{e}(N)$ that contains exactly $|N|$ free edges. The induction argument allows us to conclude that it is also true for any $c \in \tilde{\Gamma}_{P^{*}}^{e}(N)$.

Part 4: $y(N, \tilde{c})=y^{a}(N, \tilde{c})$ for all $\tilde{c} \in \tilde{\Gamma}_{P}^{e}$, the set of elementary matrices generating agent connection problems and such that the free edges constitute one connected component $N$ (but not necessarily a tree).

Parts 2 and 3 covers the cases where the connected component is $N$ and the free edges constitute a tree or a free cycle that covers all members of $N$. We cover the remaining cases. We focus on the cost share of agent $i \in N$. There are two possibilities:

i) $i$ does not belong to a free cycle

In that case, proceed exactly as in part 2: using Weak Problem Separation, Group Independence and Anonymity, "cut off" the branches attached to $i$ until that agent is left alone, allowing to find the link between $y_{i}(N, c)$ and $y_{i}\left(\{i\}, c^{\{i\}}\right)$, which is trivially equal to 1 .

ii) $i$ belongs to a free cycle. Let $S \subset N$ be such that $i \in S$, there exists a free cycle that includes all members of $S$ and there does not exists a free cycle that includes all members of $T$ for all $T \supset S$.

We then proceed as in part 2: using Weak Problem Separation, Group Independence and Anonymity, we "cut off" the branches attached to $S$ until that coalition stands alone, allowing to find the link between $y_{i}(N, c)$ and $y_{i}\left(S, c^{S}\right)$. Since $c^{S} \in \tilde{\Gamma}_{P^{*}}^{e}(S)$, by part 3 all solutions satisfying the properties coincide on $c^{S}$ and thus also coincide on $c$.

Part 5: $y(N, \tilde{c})=y^{a}(N, \tilde{c})$ for all $\tilde{c} \in \tilde{\Gamma}^{e}$, the set of elementary matrices generating agent connection problems.

Elements of $\tilde{\Gamma}^{e} \backslash \tilde{\Gamma}_{P}^{e}$ are such that we can partition $N$ into $N^{1}, . ., N^{K}$ such that that i) there exists a free path $p_{i j}$ for all $i, j \in N^{k}, k=1, \ldots, K$ and ii) there does not exist a free path $p_{l m}$ for all $l \in N^{k}$ and $m \in N^{k^{\prime}}$ if $k \neq k^{\prime}$. If a solution $\bar{y}$ satisfies Group Independence, $\bar{y}_{i}(N, c)=\bar{y}_{i}\left(N^{k}, c^{N^{k}}\right)$ for all $i \in N^{k}$, all $k \in\{1, \ldots, K\}$. Clearly, $c^{N^{k}} \in \tilde{\Gamma}_{P}^{e}$. Therefore, since, by Part $4, y(N, \tilde{c})=y^{a}(N, \tilde{c})$ for all $\tilde{c} \in \tilde{\Gamma}_{P}^{e}$, we also have that $y(N, \tilde{c})=y^{a}(N, \tilde{c})$ for all $\tilde{c} \in \tilde{\Gamma}^{e}$.

Part 6: $y(N, c)=y^{a}(N, c)$ for all $c \in \bar{\Gamma}^{e}$, the set of elementary matrices without irrelevant edges.

If $c \in \bar{\Gamma}^{e}(N)$ is such that $\min _{i \in N} c_{0 i}=1$, then $c \in \tilde{\Gamma}^{e}$ and by part $5, y(N, \tilde{c})=y^{a}(N, \tilde{c})$. Suppose that $\min _{i \in N} c_{0 i}=0$. If $C(N, c)=0$ and a solution $\bar{y}$ satisfies Weak Problem Separation, we have 
$\bar{y}(N, c)=\bar{y}_{i}(N, \hat{c})+\bar{y}_{i}(N, \tilde{c})-\bar{y}_{i}(N, \dot{c})$, with $\hat{c}, \dot{c} \in \hat{\Gamma}^{e}$ and $\tilde{c} \in \tilde{\Gamma}^{e}$. By Parts 1 and $5, y$ and $y^{a}$ coincide on $\hat{\Gamma}^{e}$ and $\tilde{\Gamma}^{e}$. Therefore, they also coincide for $c \in \bar{\Gamma}^{e}$ such that $C(N, c)=0$. Suppose that $\min _{i \in N} c_{0 i}=0$ but $C(N, c)>0$. Then, we can partition $N$ into $N^{1}, . ., N^{K}$ such that that i) there exists a free path $p_{i j}$ in $N$ for all $i, j \in N^{k}, k=1, \ldots, K$ and ii) there does not exist a free path $p_{l m}$ in $N$ for all $l \in N^{k}$ and $m \in N^{k^{\prime}}$ if $k \neq k^{\prime}$. If a solution $\bar{y}$ satisfies Group Independence, $\bar{y}_{i}(N, c)=\bar{y}_{i}\left(N^{k}, c^{N^{k}}\right)$ for all $i \in N^{k}$, all $k \in\{1, \ldots, K\}$. For all $k$, it is clear that either $c^{N^{k}} \in \bar{\Gamma}^{e}(N)$ is such that $\min _{i \in N^{k}} c_{0 i}=1$ or $C\left(N^{k}, c^{N^{k}}\right)=0$. By the results above, we thus have that $y$ and $y^{a}$ coincide on all $c \in \bar{\Gamma}^{e}(N)$ such that $\min _{i \in N} c_{0 i}=0$ but $C(N, c)>0$. Altogether, we have that $y(N, c)=y^{a}(N, c)$ for all $c \in \bar{\Gamma}^{e}$.

Part 7: $y(N, c)=y^{a}(N, c)$ for all $c \in \Gamma$, the set of all cost matrices.

Consider $c \in \Gamma^{e}$. If a solution $\bar{y}$ satisfies Independence of Irrelevant Edges, $\bar{y}(N, c)=\bar{y}(N, \bar{c})$. By Part 6, $y$ and $y^{a}$ coincide on all $c \in \Gamma^{e}$, the set of all elementary cost matrices.

Consider $c \in \Gamma$. If a solution $\bar{y}$ satisfies Piecewise Linearity, $\bar{y}(N, c)=\sum_{k=1}^{q}\left(c_{e^{\sigma(k)}}-c_{e^{\sigma(k-1)}}\right) \bar{y}\left(N, b^{k}\right)$, with $\sigma$ such that $c_{e^{\sigma(1)}} \leq c_{e^{\sigma(2)}} \leq \ldots \leq c_{e^{\sigma(q)}}$. Since $b^{k} \in \Gamma^{e}$ and $y$ and $y^{a}$ coincide on $\Gamma^{e}$, we conclude that $y(N, c)=y^{a}(N, c)$ for all $c \in \Gamma$.

Since it has been shown that all solutions $y \in Y$ satisfy the six properties and that all solutions satisfying the six properties are part of $Y$, the proof is complete.

This theorem can ease implementation of cost sharing solutions. Once the participants are convinced that the properties of the theorem are acceptable, we can ask for their preferred value of $a$ in Example 1 and obtain the corresponding cost sharing solution.

\section{New characterizations of the Folk and Kar solutions}

Quite obviously, the folk and Kar solutions are in $Y$, respectively, when $a=0$ and $a=\frac{1}{2}$. We provide new characterizations for these solutions by adding additional properties to those presented in the previous section.

The first property we add is the usual Core Selection property, which assures stability, as no group has incentives to secede and realize the project on its own. Core notions go back to Gillies (1953). Core Selection has been used in Bogomolnaia and Moulin (2010) to characterize the folk solution.

Core Selection: For any mest problem $(N, c)$ and all $S \subseteq N, \sum_{i \in S} y_{i}(N, c) \leq C(S, c)$.

Theorem 2 A solution y satisfies Weak Problem Separation, Group Independence, Piecewise Linearity, Anonymity, Independence of Irrelevant Edges, Weak Equal Treatment and Core Selection if and only if $y$ is the folk solution. ${ }^{7}$

Proof. Lemma A.1 shows that the folk solution satisfies Weak Problem Separation, Group Independence, Piecewise Linearity, Anonymity, Independence of Irrelevant Edges and Weak Equal Treatment. Lemma A.3 shows that the folk solution satisfies Core Selection and that the Kar solution does not satisfy it.

Theorem 1 shows that if a solution satisfies Weak Problem Separation, Group Independence, Piecewise Linearity, Anonymity, Independence of Irrelevant Edges and Weak Equal Treatment, then it can be written as $y^{a}=2 a\left(y^{k}-y^{f}\right)+y^{f}$, with $a \in \mathbb{R}$. Since the Kar solution does not satisfy Core Selection, it is easy to see that any solution that puts a non-zero weight on the Kar solution will not satisfy Core Selection. Therefore, we must have $a=0$ and $y=y^{f}$.

If we compare this with other characterizations of the folk solution, we see for instance that like the characterization found in Bogomolnaia and Moulin (2010), we use Piecewise Linearity and Core Selection, and Anonymity instead of Symmetry. While they use Reductionism, that says that cost shares should only depend on the irreducible cost matrix, we use Weak Problem Separation, Group Independence, Weak Equal Treatment and Independence of Irrelevant Edges. This shows how strong

\footnotetext{
${ }^{7}$ We can also replace Core Selection by Population Monotonicity, given that the former is implied by the latter and that the folk solution satisfies both (Bergantinos and Vidal-Puga (2007a)). Population Monotonicity requires that no agent be made worse off when agents join the coalition.
} 
the Reductionism property is. Similarly, Bergantinos and Vidal-Puga (2009) characterize the folk solution with Symmetry, a stronger version of Piecewise Linearity and Separability, a stronger version of Group Independence that states that if $C(S, c)+C(N \backslash S, c)=C(N, c)$ then we can compute cost shares of $S$ and $N \backslash S$ independently. This is stronger than Group Independence as it applies to cases where $C(R, c)+C(T, c)>C(R \cup T, c)$ where $R \subset S$ and $T \subseteq N \backslash S$, whereas the Group Independence property only applies when two groups are completely disjoint. It seems like this strengthening of the Group Independence axiom is significant, because, in our case, we need to compensate by adding Weak Problem Separation, Weak Equal Treatment, Independence of Irrelevant Edges and Core Selection.

The stronger version of Problem Separation is satisfied by the Kar solution. It turns out that it is the only solution in the family described in the previous section that satisfies it.

Theorem 3 A solution y satisfies Problem Separation, Group Independence, Piecewise Linearity, Anonymity, Weak Equal Treatment and Independence of Irrelevant Edges if and only if $y$ is the Kar solution.

Proof. Lemma A.1 shows that the Kar solution satisfies Weak Problem Separation, Group Independence, Piecewise Linearity, Anonymity, Independence of Irrelevant Edges and Weak Equal Treatment. Lemma A.3 shows that the Kar solution satisfies Problem Separation and that the folk solution does not.

Theorem 1 shows that if a solution satisfies Weak Problem Separation, Group Independence, Piecewise Linearity, Anonymity, Independence of Irrelevant Edges and Weak Equal Treatment, then it can be written as $y^{a}=2 a\left(y^{k}-y^{f}\right)+y^{f}$, with $a \in \mathbb{R}$. Since the folk solution does not satisfy Problem Separation, it is easy to see that any solution that puts a non-zero weight on the folk solution will not satisfy Problem Separation. Therefore, we must have $a=\frac{1}{2}$ and $y=y^{k}$.

The only characterization of the Kar solution appears in Kar (2002). It uses a weaker version of Group Independence (strict inequalities) and Absence of Cross Subsidization, which together act as the version of Group Independence used here. They are used with the strong version of Equal Treatment, that says that if the cost of any edge $(i, j)$ changes, then the cost shares of agents $i$ and $j$ should change by the same amount. This strong property is replaced in our characterization by its restricted version, Weak Equal Treatment, and Piecewise Linearity, Anonymity, Independence of Irrelevant Edges and Problem Separation.

We now consider two properties making agents responsible for where they are located in the network. Bogomolnaia and Moulin (2010) consider similar properties, however restricted to cost matrices where the edges connecting agents to the source are strictly more expensive than other edges. They are also discussed in Trudeau (2012b), where a compromise between the Kar and folk solutions is introduced.

Strict Cost Monotonicity: For any mcst problems $(N, c)$ and $\left(N, c^{\prime}\right)$ such that $c_{i j}^{\prime}<c_{i j} \leq$ $\max \left\{c_{0 i}, c_{0 j}\right\}$ and $c_{e}^{\prime}=c_{e}$ else, we have $y_{k}\left(N, c^{\prime}\right)<y_{k}(N, c)$ for $k \in\{i, j\}$.

Strict Cost Monotonicity says that for a relevant edge $(i, j)$, if its cost decreases and the cost of all other edge stay the same, then both agents $i$ and $j$ see their cost allocations strictly decrease.

Strict Ranking: For any mcst problem $(N, c)$ such that $c_{i k} \leq c_{j k}$ for all $k \in N_{0} \backslash\{i, j\}$ and $c_{i l}<c_{j l}$ for some $l \in N_{0} \backslash\{i, j\}$, with $c_{i l}<\max \left\{c_{0 i}, c_{0 l}\right\}$, we have $y_{i}(N, c)<y_{j}(N, c)$.

Strict Ranking says that the location of agent $i$ is strictly better than the location of agent $j$, then the cost allocation of $i$ is strictly less than the cost allocation of $j$.

Lemma A.4 in appendix shows that members of the family that satisfy these properties are such that $a>0$. Therefore, both eliminate the folk solution.

While $a$ can take any value in $\mathbb{R}$, if $a<0$, then in Example 1 agent one has a cost share that is higher than 0 , his stand-alone cost. Similarly, if $a>1$, it is agent 2 that has a cost share that is higher than his stand-alone cost. In those cases, agents would be better off not cooperating with each other. This weak stability property, called Individual Rationality (or Stand-Alone property), is formally defined as follows.

Individual Rationality: For any mcst problem $(N, c)$ and $i \in N, y_{i}(N, c) \leq C(\{i\}, c)=c_{0 i}$.

Lemma A.4 in appendix shows formally that this property is satisfied if and only if $a \in[0,1]$. A value of $a=1$, that generates a solution $y^{1}=2 y^{k}-y^{f}$, means that in Example 1 we give all of the 
surplus created to agent 1, who has the better location. By opposition, the folk solution gives all of the surplus to agent 2, who helps create the surplus by demanding to be connected to the source. Therefore, the main result of the paper can be restated as follows, providing a new justification for the Kar solution: the set of rules corresponding to the convex combination of the folk solution and $y^{1}$ are the only ones satisfying Weak Problem Separation, Group Independence, Piecewise Linearity, Anonymity, Weak Equal Treatment, Independence of Irrelevant Edges and Individual Rationality. Moreover, the average of these rules is the Kar solution.

\section{Appendix}

Lemma A.1 All $y \in Y$ as defined in Theorem 1 satisfy Weak Problem Separation, Group Independence, Piecewise Linearity, Anonymity, Weak Equal Treatment and Independence of Irrelevant Edges.

Proof. Notice that the weight on the Kar solution is $2 a$ while the weight on the folk solution is $1-2 a$. Let $\theta=2 a$ and rewrite the family of solutions as $\theta y^{k}+(1-\theta) y^{f}$. The family of solutions is a weighted sum of the Kar and folk solutions, with the weights summing to one, but the weights being possibly negative. Since both solutions are budget balanced and the weights sum to one, all of the solutions of Theorem 1 are also budget balanced.

Since the Kar and folk solutions are part of the family, we first show that they satisfy all properties. This result then trivially extends to solutions $y=2 a\left(y^{k}-y^{f}\right)+y^{f}$, the weighted sums of these two solutions.

We start with the Kar solution. Symmetry is proven in Bergantinos and Vidal-Puga (2007a); Anonymity carries over easily. Group Independence is proven in Kar (2002) (the proof easily extends when we remove strict inequality). Piecewise Linearity is proven in Bogomolnaia and Moulin (2010).

We show that the Kar solution satisfies the stronger property of Problem Separation: By Lemma $1, C(S, c)=C(S, \hat{c})+C(S, \tilde{c})-C(S, \dot{c})$. By the properties of the Shapley value, $y^{k}(N, c)=y^{k}(N, \hat{c})+$ $y^{k}(N, \tilde{c})-y^{k}(N, \dot{c})$.

Weak Equal Treatment: It is implied by Equal Treatment (Kar (2002)).

Independence of Irrelevant Edges: Clearly, $C(S, c)=C(S, \bar{c})$ for all $S \subseteq N$. Therefore, $y^{k}(N, c)=$ $y^{k}(N, \bar{c})$.

Next, we show that the folk solution satisfies all of these properties.

Bergantinos and Vidal-Puga (2007a) show that it satisfies Symmetry; Anonymity carries over easily. They also show that it satisfies Reductionism $\left(y(N, c)=y\left(N, c^{*}\right)\right)$ and Separability, which says that if $C(S, c)+C(N \backslash S, c)=C(N, c)$, then $y_{i}(N, c)=y_{i}\left(S, c^{S}\right)$ if $i \in S$.

Reductionism clearly implies Independence of Irrelevant Edges, as the irreducible edges cannot have any impact on the irreducible matrix. It also clearly implies Weal Equal Treatment.

Separability is clearly a stronger property than Group Independence.

Bergantinos and Vidal-Puga (2009) show that it satisfies Restricted Additivity, which says that if $c, c^{\prime}$ have an mcst $t^{*}$ in common and that the edges of that $t^{*}$ have a common order of its edges from cheapest to most expensive, then $y\left(N, c+c^{\prime}\right)=y(N, c)+y\left(N, c^{\prime}\right)$. It clearly implies Piecewise Linearity. It also implies Weak Problem Separation. To see this, notice that $c+\dot{c}=\tilde{c}+\hat{c}$ and that under the assumption that $c \in \bar{\Gamma}(N)$, if $c_{e} \leq \min _{i \in N} c_{0 i}$ for all $e \in t^{*}(c)$, all $t^{*} \in T^{*}(c)$, they all have a common mcst $t$ that has a common order of its edges from cheapest to most expensive. Then, Restricted Additivity implies that $y^{f}(N, c)+y^{f}(N, \dot{c})=y^{f}(N, \hat{c})+y^{f}(N, \tilde{c})$.

It is easy to show that Weak Problem Separation, Group Independence, Piecewise Linearity, Anonymity, Weak Equal Treatment and Independence of Irrelevant Edges extend to any solution $y^{a}=2 a\left(y^{k}-y^{f}\right)+y^{f}$ since they are satisfied by both $y^{k}$ and $y^{f}$.

Lemma A.2 The six properties of Theorem 1 are independent.

Proof. Since $N$ is fixed throughout, we define a solution as $y(c)$ instead of $y(N, c)$. 
i) Weak Problem Separation: Define $c^{1}$ as follows: for each $i \in N$, look at cycles that go through 0 and $i$. Let $F_{0 i}$ be the set of all such cycles. Let $c_{0 i}^{1}=\min \left\{c_{0 i}, \min _{f \in F_{0 i}} \max _{e \in f} c_{e}\right\}$ and $y^{i}(c)=$ $\operatorname{Sh}\left(C\left(\cdot, c^{1}\right)\right)$.

It clearly satisfies Piecewise Linearity, Weak Equal Treatment, Anonymity, Group Independence and Independence of Irrelevant Edges. It fails Weak Problem Separation. To see this, consider a 3 -player example such that $c_{03}=c_{23}=1$ and $c_{e}=0$ else. We have that $c^{1}=c$, yielding $y^{i}(c)=$ $\left(-\frac{1}{2}, 0, \frac{1}{2}\right)$. Now, divide the problem in its source connection and agent connection problems. We have that $\hat{c}_{03}^{1}=0$ while $\hat{c}_{03}=1$ and $\tilde{c}=\tilde{c}^{1}$. Therefore, we have $y^{i}(\hat{c})=(0,0,0)$ and $y^{i}(\tilde{c})=\left(0, \frac{1}{2}, \frac{1}{2}\right)$. Thus, $y^{i}(c) \neq y^{i}(\hat{c})+y^{i}(\tilde{c})-\left(\frac{1}{3}, \frac{1}{3}, \frac{1}{3}\right)$.

ii) Group Independence. Let $y_{j}^{i i}(c)=\frac{C(N, c)}{n}$ for all $j \in N$. It is easy to see that it satisfies Piecewise Linearity, Weal Equal Treatment, Anonymity, Independence of Irrelevant Edges and Weak Problem Separation. Suppose a two-player example where $c_{01}=3, c_{02}=c_{12}=5$. By Group Independence, we should have $y=(3,5)$ but $y^{i i}=(4,4)$.

iii) Piecewise Linearity. Let $\gamma(c)$ be the cost of the most expensive edge in an mcst of $c$. If we order edges from cheapest to most expensive, then rank $K^{\gamma}$ is such that $c_{e} \leq \gamma(c)$ if the rank of $e$ is below $K^{\gamma}$ and $c_{e}>\gamma(c)$ if the rank of $e$ is above $K^{\gamma}$. Let $y^{i i i}(c)=\left\{\begin{array}{c}y^{k}(c) \text { if } c_{e} \leq \min _{i \in N} c_{0 i} \text { for all } e \in t^{*}(c) \text {, all } t^{*} \in T^{*}(c) \\ \sum_{k=1}^{K^{\gamma}}\left(c_{e^{\sigma(k)}-} c_{e^{\sigma(k-1)}}\right) y^{k}\left(b^{k}\right) \text { else }\end{array}\right\}$. It clearly fails Piecewise Linearity, as between ranks $K^{\gamma}$ and $q, y^{k}\left(b^{k}\right)$ is typically not equal to $(0,0, \ldots, 0)$. It satisfies Weak Equal Treatment as the Kar solution satisfies it (for the first segment). In the second segment, if an eligible edge is such that its cost is above $\gamma(c)$, a reduction in cost won't have any effect. Anonymity, Group Independence and Independence of Irrelevant Edges are clearly satisfied. Weak Problem Separation only applies in the first part, where it is satisfied by the Kar solution.

iv) Anonymity. Order agents randomly and let $y_{1}^{i v}(c)=C\left(\{1\}, c^{*}\right)$ and $y_{j}^{i v}(c)=C\left(\{1, \ldots, j\}, c^{*}\right)-$ $C\left(\{1, \ldots, j-1\}, c^{*}\right)$ for all $j=2, \ldots, n$. It is easy to see that it satisfies Piecewise Linearity, Weak Problem Separation, Group Independence and Independence of Irrelevant Edges. It satisfies Weak Equal Treatment because edges on which it applies do not affect the irreducible cost matrix. Suppose that $c_{01}=c_{02}=3, c_{12}=1$ and $c_{1 j}=c_{2 j}$ else. Then, $y_{1}^{i v}=3$ and $y_{2}^{i v}=1$. It fails Anonymity.

v) Weak Equal Treatment. For any $c \in \hat{\Gamma}^{e}$ and $c \in \tilde{\Gamma}^{e} \backslash \tilde{\Gamma}_{P^{*}}^{e}$, let $y^{v}(c)=y^{k}(c)$. For $c \in \tilde{\Gamma}_{P^{*}}^{e}$, let $y^{v}(c)=y^{f}(c)$. Through Piecewise Linearity, Independence of Irrelevant Edges and Weak Problem Separation, extend the definition of $y^{v}$ to any $c \in \Gamma$. By definition, Piecewise Linearity, Independence of Irrelevant Edges and Weak Problem Separation are satisfied. Since both the Kar and the folk solutions satisfy Anonymity and Group Independence, so does $y^{v}$. It clearly fails Weak Equal Treatment: Suppose that we start from $c \in \tilde{\Gamma}^{e} \backslash \tilde{\Gamma}_{P^{*}}^{e}$, and that changing the cost of $c_{i j}$ from 1 to 0 creates a free cycle, we obtain a cost matrix in $\tilde{\Gamma}_{P^{*}}^{e}$. Going from the folk to the Kar solution will typically affect agents $i$ and $j$ differently.

vi) Independence of Irrelevant Edges: We define $y^{v i}$ as follows on elementary matrices. For $c \in \bar{\Gamma}^{e}$, we have that $y^{v i}(c)=y^{k}(c)$. Take $c \in \bar{\Gamma}^{e}$ such that $c_{0 i}=c_{0 j}=c_{i j}=0$. Let $c^{\prime}$ be such that $c_{i j}^{\prime}=1$ and $c_{e}^{\prime}=c_{e}$ else. Let $N_{i}(c)$ be the set of players to which $i$ can connect freely to without going through the source. Define $y^{v i}\left(c^{\prime}\right)$ as follows:

$$
y_{l}^{v i}\left(c^{\prime}\right)=\left\{\begin{array}{c}
y_{l}^{v i}(c)+b \text { for } l=i, j, \text { with } b>0 \\
y_{l}^{v i}(c)-\frac{2 b}{\left|N_{i}(c)\right|} \text { for } l \in N_{i}^{*}(c) \backslash\{i, j\} . \\
y_{l}^{v i}(c) \text { else }
\end{array} .\right.
$$

Having defined all cost shares for elementary matrices with one irrelevant edge in this manner, we proceed in the same manner for all elementary matrices with 2 irrelevant edges, then recursively for any number of irrelevant edges. We then extend to any $c \in \Gamma$ through Piecewise Linearity.

By definition, $y^{v i}$ satisfies Piecewise Linearity. It is easy to see that it satisfies Anonymity and Weak Equal Treatment. Since Weak Problem Separation applies only to matrices without irrelevant edges, only its properties on $\bar{\Gamma}^{e}$ matter. Since it is equal to the Kar solution for those matrices, Weak Problem Separation is satisfied. Group Independence is satisfied since the difference between $y_{l}^{v i}\left(c^{\prime}\right)$ 
and $y_{l}^{v i}(c)$ depends if you are freely connected to $i$ and $j$ or not. It is easy to see that Independence of Irrelevant Edges is not satisfied.

The following table summarizes the results of the previous two lemmas, with "+" meaning that the property is satisfied and "-" that it is not.

Satisfaction of the properties characterizing $Y$ by the folk and Kar solutions as well as solutions defined in Lemma A.2

\begin{tabular}{|c|c|c|c|c|c|c|}
\hline & WPS & GI & PL & ANON & WET & IIE \\
\hline$y^{f}$ & + & + & + & + & + & + \\
\hline$y^{k}$ & + & + & + & + & + & + \\
\hline$y^{i}$ & - & + & + & + & + & + \\
\hline$y^{i i}$ & + & - & + & + & + & + \\
\hline$y^{i i i}$ & + & + & - & + & + & + \\
\hline$y^{i v}$ & + & + & + & - & + & + \\
\hline$y^{v}$ & + & + & + & + & - & + \\
\hline$y^{v i}$ & + & + & + & + & + & \\
\hline
\end{tabular}

Lemma A.3 i) The folk solution satisfies Core Selection but it does not satisfy Problem Separation. ii) The Kar solution satisfies Problem Separation but it does not satisfy Core Selection.

Proof. i) Bergantinos and Vidal-Puga (2007a) show that the folk solution satisfies Core Selection.

Take $c \in \bar{\Gamma}^{e}$ such that $c_{0 i}=c_{0 j}=c_{i j}=0$ and $c_{e}=1$ else. We have $y_{l}^{f}(N, c)=0$ if $l=\{i, j\}$ and 1 else. Also, we have $y_{l}^{f}(N, \hat{c})=0$ for all $l \in N$ and $y_{l}^{f}(N, \tilde{c})=\frac{1}{2}$ if $l=\{i, j\}$ and 1 else. Therefore, $y_{l}^{f}(N, \hat{c})+y_{l}^{f}(N, \tilde{c})-\frac{1}{|N|} \neq y_{l}^{f}(N, c)$. Problem Separation is not satisfied.

ii) Problem Separation was proved in Lemma A.1.

Consider the problem $c$ such that $c_{j k}=0$ for all $j, k \in N, c_{0 j}=0$ for all $j \in N \backslash\{i\}$ and $c_{0 i}=1$, with $|N|>2$. Then, $y_{i}^{k}(N, c)=\frac{1}{|N|}$ and $y_{j}^{k}(N, c)=-\frac{1}{|N|(|N|-1)}$ for all $j \in N \backslash\{i\}$. We have $C(S, c)=0$ for all $S \neq\{i\}$. Take $l \in N \backslash\{i\}$. We obtain $y_{i}(N, c)+y_{l}(N, c)=\frac{(|N|-2)}{|N|(|N|-1)}>0$. The Kar solution fails to satisfy Core Selection.

Lemma A.4 Suppose that $y^{a}(N, c)=2 a y^{k}(N, c)+(1-2 a) y^{f}(N, c)$ with $a \in \mathbb{R}$. We have that:

i) $y^{a}(N, c)$ satisfies Individual Rationality if and only if $a \in[0,1]$.

ii) $y^{a}(N, c)$ satisfies Strict Cost Monotonicity if and only if $a>0$.

iii) $y^{a}(N, c)$ satisfies Strict Ranking if and only if $a>0$.

Proof. i) Example 1 shows that Individual Rationality is not satisfied if $a<0$ or $a>1$. We show that $a \in[0,1]$ is a sufficient condition for Individual Rationality.

For $a \in\left[0, \frac{1}{2}\right], y^{a}$ is a convex combination of the Kar and folk solutions, who both satisfy Individual Rationality, $y^{a}$ also satisfies it.

For $a \in\left[\frac{1}{2}, 1\right]$, we have that $y^{a}(N, c)=y^{k}(N, c)+b\left(y^{k}(N, c)-y^{f}(N, c)\right)$ with $b=2 a-1 \geq 0$.

For any $c \in \Gamma^{e}$, if $c_{0 i}=0$, we have that $y_{i}^{f}(N, c)=0$ (since it satisfies Individual Rationality and Non-Negativity) while $y_{i}^{k}(N, c) \leq 0$ (by Individual Rationality). Therefore, $y^{k}(N, c)-y^{f}(N, c) \leq 0$, which implies that $y_{i}^{a}(N, c) \leq 0=c_{0 i}$ for any $a \in\left[\frac{1}{2}, 1\right]$.

Suppose that $c_{0 i}=1$ and $Z_{i}(c)=\emptyset$. Then it is easy to see that $y_{i}^{k}(N, c)=y_{i}^{f}(N, c)=1$, as agent $i$ has no free path connecting him to anybody else. Thus, $y_{i}^{a}(N, c)=1=c_{0 i}$.

Suppose that $c_{0 i}=1$ and $j \in Z_{i}(c)$. Then, we have that $y_{i}^{k}(N, c) \leq \frac{1}{2}$. To see this notice that when agent $i$ comes in after agent $j$, his incremental cost is zero, as he can connect freely to agent $j$. There is a probability $\frac{1}{2}$ that he comes in after agent $j$. Any other incremental cost cannot be larger than 1 . We thus have that $y_{i}^{a}(N, c) \leq \frac{1}{2}+b\left(\frac{1}{2}-y_{i}^{f}(N, c)\right)$. By the Non-Negativity of the folk solution, we have that $y_{i}^{a}(N, c) \leq \frac{1}{2}+\frac{b}{2}$. For any $b \leq 1$, we have $y_{i}^{a}(N, c) \leq 1=c_{0 i}$. We have that $b \leq 1$ if and only if $a \leq 1$. 
Therefore, Individual Rationality is satisfied for all $c \in \Gamma^{e}$ if $a \in[0,1]$.

For $c \in \Gamma$, we can apply Piecewise Linearity. We have $y_{i}^{a}(N, c)=\sum_{k=1}^{q}\left(c_{e^{\sigma(k)}}-c_{e^{\sigma(k-1)}}\right) y_{i}^{a}\left(N, b^{k}\right)$ with all $b^{k} \in \Gamma^{e}$. Therefore, $y_{i}^{a}\left(N, b^{k}\right) \leq b_{0 i}^{k}$ for all $k$. Since $c_{0 i}=\sum_{k=1}^{q}\left(c_{e^{\sigma(k)}}-c_{e^{\sigma(k-1)}}\right) b_{0 i}^{k}$ and all the weights are positive, we have $y_{i}^{a}(N, c) \leq c_{0 i}$. Individual Rationality is satisfied for all $c \in \Gamma$ if $a \in[0,1]$.

ii) Suppose that $c_{i j} \leq \max \left\{c_{0 i}, c_{0 j}\right\}$ and $c, c^{\prime}$ such that $c_{i j}^{\prime}<c_{i j}$ and $c_{e}^{\prime}=c_{e}$ else.

Define $\Delta y_{l}^{k}=y_{l}^{k}(N, c)-y_{l}^{k}\left(N, c^{\prime}\right)$ and $\Delta y_{l}^{f}=y_{l}^{f}(N, c)-y_{l}^{f}\left(N, c^{\prime}\right)$. By the properties of the Kar and folk solutions, $\Delta y_{l}^{k}>0$ and $\Delta y_{l}^{f} \geq 0$ for $l \in\{i, j\}$. We have Strict Cost Monotonicity if $2 a \Delta y_{l}^{k}+(1-2 a) \Delta y_{l}^{f}>0$ for $l \in\{i, j\}$.

For the following, suppose that $l \in\{i, j\}$. Suppose that $\Delta y_{l}^{f}=0$ (which happens when $c_{i j}^{\prime} \geq \bar{c}_{i j}$ ). We have $2 a \Delta y_{l}^{k}>0$ if and only if $a>0$.

We need to show that $2 a \Delta y_{l}^{k}+(1-2 a) \Delta y_{l}^{f}>0$ when $\Delta y_{l}^{f}>0$. If $0<a \leq \frac{1}{2}$, we have $2 a>0$ and $1-2 a \geq 0$. Combined with $\Delta y_{l}^{k}>0$ and $\Delta y_{l}^{f} \geq 0$, it assures that $2 a \Delta y_{l}^{k}+(1-2 a) \Delta y_{l}^{f}>0$.

We have that $\Delta y_{l}^{k} \geq \Delta y_{l}^{f}$. To see this, notice that the reduction of the cost on $(i, j)$ reduces $C(S, c)$ for all coalitions $S \supseteq\{i, j\}$ that uses that edge. The reduction of the cost on $(i, j)$, if it modifies the irreducible matrix, will change the value of $\bar{c}_{i j}$ but potentially of some other edge $\bar{c}_{e}$, meaning that $\bar{C}(S, c)$ can decrease for some $S \nsupseteq\{i, j\}$. Therefore, if $a>\frac{1}{2}$, we have $2 a \Delta y_{l}^{k}+(1-2 a) \Delta y_{l}^{f} \geq$ $2 a \Delta y_{l}^{k}+(1-2 a) \Delta y_{l}^{k}=\Delta y_{l}^{k}>0$.

Therefore, Strict Cost Monotonicity is satisfied if and only if $a>0$.

iii) Suppose that $c_{i k} \leq c_{j k}$ for all $k \in N_{0} \backslash\{i, j\}$ and $c_{i l}<c_{j l}$ for some $l \in N_{0} \backslash\{i, j\}$, with $c_{i l}<\max \left\{c_{0 i}, c_{0 l}\right\}$.

Define $\Delta y_{i j}^{k}=y_{j}^{k}-y_{i}^{k}$ and $\Delta y_{i j}^{f}=y_{j}^{f}-y_{i}^{f}$. By the properties of the Kar and folk solutions, $\Delta y_{i j}^{k}>0$ and $\Delta y_{i j}^{f} \geq 0$. We have Strict Ranking if $2 a \Delta y_{i j}^{k}+(1-2 a) \Delta y_{i j}^{f}>0$. Suppose that $\Delta y_{i j}^{f}=0$ (which happens when $\bar{c}_{i l}=\bar{c}_{j l}$ ). We have $2 a \Delta y_{i j}^{k}>0$ if and only if $a>0$.

We need to show that $2 a \Delta y_{i j}^{k}+(1-2 a) \Delta y_{i j}^{f}>0$ when $\Delta y_{i j}^{f}>0$. If $0<a \leq \frac{1}{2}$, we have $2 a>0$ and $1-2 a \geq 0$. Combined with $\Delta y_{i j}^{k}>0$ and $\Delta y_{i j}^{f} \geq 0$, it assures that $2 a \Delta y_{i j}^{k}+(1-2 a) \Delta y_{i j}^{f}>0$.

We have that $\Delta y_{i j}^{k} \geq \Delta y_{i j}^{f}$. To see this, start with a case where $c_{i k}=c_{j k}$ for all $k \in N_{0} \backslash\{i, j\}$. Solutions satisfying Anonymity, like the Kar and folk solutions, will be such that $y_{i}=y_{j}$. Then, reduce the cost of some edges $(i, k)$. As $C(S, c)$ can decrease only if $\{i, k\} \subseteq S, i$ and $k$ are the main beneficiaries. If the reductions change the irreducible matrix, in particular if it reduces the cost of some edges $(j, l)$, then part of the savings are passed on to other agents, including agent $j$. Therefore, if $a>\frac{1}{2}$. we have $2 a \Delta y_{i j}^{k}+(1-2 a) \Delta y_{i j}^{f} \geq 2 a \Delta y_{i j}^{k}+(1-2 a) \Delta y_{i j}^{k}=\Delta y_{i j}^{k}>0$.

Therefore, Strict Ranking is satisfied if and only if $a>0$.

Acknowledgement 1 Special thanks to an anonymous referee who went the extra mile to improve the proof of the main theorem. I also thank Hervé Moulin, Yuntong Wang and participants of the 2010 Canadian Economic Theory Conference for useful comments. Financial support from the Social Sciences and Humanities Research Council of Canada (SSHRC) is gratefully acknowledged.

\section{References}

Bergantinos G, Kar A (2010) On obligation rules for minimum cost spanning tree problems. Games and Economic Behavior 69:224-237

Bergantinos G, Vidal-Puga J (2007a) A fair rule in minimum cost spanning tree problems. Journal of Economic Theory 137:326-352

Bergantinos G, Vidal-Puga J (2007b) The optimistic tu game in minimum cost spanning tree problems. International Journal of Game Theory 36:223-239 
Bergantinos G, Vidal-Puga J (2009) Additivity in minimum cost spanning tree problems. Journal of Mathematical Economics 45:38-42

Bergantinos G, Vidal-Puga J (2010) Realizing fair outcomes in minimum cost spanning tree problems through non-cooperative mechanisms. European Journal of Operational Research 201:811-820

Bogomolnaia A, Moulin H (2010) Sharing the cost of a minimal cost spanning tree: Beyond the folk solution. Games and Economics Behavior 69:238-248

Branzei R, Moretti S, Norde H, Tijs S (2004) The p-value for cost sharing in minimal cost spanning tree situations. Theory and Decision 56:47-61

Feltkamp V, Tijs S, Muto S (1994) On the Irreducible Core and the Equal Remaining Obligations Rule of Minimum Cost Spanning Extension Problems. Tilburg University CentER Discussion Paper 94106

Gillies DB (1953) Some theorems on n-person games. Ph.D. Thesis, Department of Mathematics, Princeton University

Kar A (2002) Axiomatization of the Shapley value on minimum cost spanning tree games. Games and Economic Behavior 38:265-277

Moulin H (1988) Axioms of Cooperative Decision Making. Cambridge University Press

Norde H, Moretti S, Tijs S (2004) Minimum cost spanning tree games and population monotonic allocation schemes. European Journal of Operational Research 154:84-97

Shapley LS (1953) A Value for n-person Games, in: H.W. Kuhn and A.W. Tucker (Eds), Contributions to the Theory of Games, volume II. Princeton University Press, NJ

Tijs S, Branzei R, Moretti S, Norde H (2006) Obligation rules for msct situations and their monotonicity properties. European Journal of Operational Research 175:121-134

Trudeau C (2012a) Characterizations of the Kar and folk solutions for minimum cost spanning tree problems. International Game Theory Review, forthcoming

Trudeau C (2012b) A new stable and more responsive cost sharing solution for minimum cost spanning tree problems. Games and Economic Behavior 75:402-412

Winter E (2002) The Shapley Value, in: R.J. Aumann and S. Hart (Eds.), The Handbook of Game Theory. North-Holland 